\title{
Synthetic Tau Fibrils Mediate Transmission of Neurofibrillary Tangles in a Transgenic Mouse Model of Alzheimer's-Like Tauopathy
}

\author{
Michiyo Iba, Jing L. Guo, Jennifer D. McBride, Bin Zhang, John Q. Trojanowski, and Virginia M.-Y. Lee \\ Center for Neurodegenerative Disease Research, Institute on Aging, Department of Pathology and Laboratory, University of Pennsylvania School of \\ Medicine, Philadelphia, Pennsylvania 19104
}

\begin{abstract}
Tauopathies, including Alzheimer's disease $(\mathrm{AD})$ and frontotemporal lobar degeneration with tau pathologies, are neurodegenerative diseases characterized by neurofibrillary tangles (NFTs) comprising filamentous tau protein. Although emerging evidence suggests that tau pathology may be transmitted, we demonstrate here that synthetic tau fibrils are sufficient to transmit tau inclusions in a mouse model. Specifically, intracerebral inoculation of young PS19 mice overexpressing mutant human tau (P301S) with synthetic preformed fibrils (pffs) assembled from recombinant full-length tau or truncated tau containing four microtubule binding repeats resulted in rapid induction of NFT-like inclusions that propagated from injected sites to connected brain regions in a time-dependent manner. Interestingly, injection of tau pffs into either hippocampus or striatum together with overlaying cortex gave rise to distinct pattern of spreading. Moreover, unlike tau pathology that spontaneously develops in old PS19 mice, the pff-induced tau inclusions more closely resembled AD NFTs because they were Thioflavin S positive, acetylated, and more resistant to proteinase K digestion. Together, our study demonstrates that synthetic tau pffs alone are capable of inducing authentic NFT-like tau aggregates and initiating spreading of tau pathology in a tauopathy mouse model.
\end{abstract}

\section{Introduction}

Neurodegenerative tauopathies, characterized by abnormal tau accumulations, include some of the most common neurodegenerative diseases, such as Alzheimer's disease (AD), frontotemporal lobar degeneration, progressive supranuclear palsy and corticobasal degeneration (Lee et al., 2001; Ballatore et al., 2007). Tau is a microtubule-associated protein predominantly expressed in neurons in which it stabilizes microtubules (MTs) and promotes their assembly (Witman et al., 1976; Drechsel et al., 1992). The MT-binding affinity of tau is regulated by various posttranslational modifications, including phosphorylation (Lee et al., 1991), acetylation (Min et al., 2010; Cohen et al., 2011; Irwin et al., 2012), and glycosylation (for review, see Gong et al., 2005; Ballatore et al., 2007; Martin et al., 2011). Pathological tau

\footnotetext{
Received June 1, 2012; revised 0ct. 19, 2012; accepted 0ct. 30, 2012

Author contributions: M.I. and V.M.-Y.L. designed research; M.I., J.D.M., and B.Z. performed research; M.I., J.L.G., and J.D.M. analyzed data; M.I., J.L.G., J.Q.T., and V.M.-Y.L. wrote the paper.

This work was supported by National Institutes of Health Grant AG17586 and the Marian S. Ware Alzheimer Program Cure Alzheimer's Fund, the Karen Cohen Segal and Christopher S. Segal Alzheimer Drug Discovery Initiative Fund, the Paula C. Schmerler Fund for Alzheimer's Research, the Barrist Neurodegenerative Disease Research Fund, the Eleanor Margaret Kurtz Endowed Fund, the Mary Rasmus Endowed Fund for Alzheimer's Research, Gloria J. Miller, and Dr. Arthur Peck. We thank Joshua Daniels, Ashley Kim, and Susan Leight for their assistance in mouse husbandry, Theresa Schuck, John Robinson, and Kevin Raible for their technical assistance in immunohistochemistry, and Young Baek for his computer expertise in making the heat maps.

The authors declare no competing financial interests.

Correspondence should be addressed to Virginia M.-Y. Lee, Center for Neurodegenerative Disease Research, Maloney 3, Hospital of the University of Pennsylvania, 3600 Spruce Street, Philadelphia, PA 19104-4283. E-mail: vmylee@upenn.edu.

DOI:10.1523/JNEUROSCI.2642-12.2013

Copyright $\odot 2013$ the authors $\quad 0270-6474 / 13 / 331024-14 \$ 15.00 / 0$
}

protein is hyperphosphorylated, conformationally altered, and aggregated into insoluble neurofibrillary tangles (NFTs) in perikarya as well as in processes as neuropil threads, which are the signature pathological hallmarks of tauopathies (for review, see Ballatore et al., 2007). Accumulation of possibly toxic intracellular aggregates accompanied by loss of soluble tau capable of stabilizing MTs may synergistically lead to compromised neuronal survival (for review, see Lee et al., 2011), accounting for strong correlations between NFT burden and cognitive decline in AD patients (Terry et al., 1981; Gómez-Isla et al., 1997). Moreover, the discovery of $>30$ mutations in the MAPT gene in familial frontotemporal lobar degeneration-Tau (FTLD-Tau) (Hutton et al., 1998; Spillantini et al., 1998) suggests that tau abnormalities alone can cause neurodegeneration.

In AD brains, NFT progressively accumulate in a well-defined stereotypical pattern, with pathology first emerging in entorhinal cortex, followed by hippocampus, and eventually neocortex (Braak and Braak, 1991). Recent studies in cultured cells demonstrated that exogenously supplied misfolded tau can be taken up into cells and seed the aggregation of endogenous soluble tau (Frost et al., 2009; Guo and Lee, 2011), and injections of brain lysates from diseased tauopathy transgenic $(\mathrm{Tg})$ mice into other $\mathrm{Tg}$ mice that do not develop NFTs induce tau pathology that spreads to distant neurons (Clavaguera et al., 2009), thereby providing a plausible mechanism for the presumptive spread of NFTs in AD (for review, see Braak and Del Tredici, 2011b). Similarly, brain lysates containing $\beta$-amyloid $(\mathrm{A} \beta$ ) have been implicated in cell-to-cell transmission (Meyer-Luehmann et al., 2006; Eisele et al., 2010), whereas Luk et al. (2012) and Stöhr et al. 
Table 1. Mice numbers used in the experiments

\begin{tabular}{|c|c|c|c|c|c|c|c|c|c|c|c|c|c|}
\hline \multirow[b]{3}{*}{ After injection } & \multicolumn{8}{|c|}{ Hippocampus } & \multicolumn{4}{|c|}{ Striatum and cortex } & \multirow{3}{*}{$\begin{array}{l}\text { Uninjectec } \\
\text { aged PS19 }\end{array}$} \\
\hline & \multirow[b]{2}{*}{ PBS } & \multirow{2}{*}{$\begin{array}{l}5 \mu \mathrm{g} \text { of } \\
\text { synuclein }\end{array}$} & \multicolumn{3}{|c|}{ T40/PS } & \multicolumn{3}{|c|}{$\mathrm{K} 18 / \mathrm{PL}$} & \multirow{2}{*}{$\begin{array}{l}\text { T40/PS } \\
5 \mu \mathrm{g}\end{array}$} & \multicolumn{3}{|c|}{$\mathrm{K} 18 / \mathrm{PL}$} & \\
\hline & & & $5 \mu \mathrm{g}$ & $0.5 \mu \mathrm{g}$ & $0.05 \mu \mathrm{g}$ & $5 \mu \mathrm{g}$ & $0.5 \mu \mathrm{g}$ & $0.05 \mu \mathrm{g}$ & & $5 \mu \mathrm{g}$ & $0.5 \mu \mathrm{g}$ & $0.05 \mu \mathrm{g}$ & \\
\hline 2 weeks & & & 2 & 2 & 2 & 2 & 2 & 2 & & 2 & 2 & 2 & \\
\hline 1 month & & & 3 & 2 & 2 & 5 & 2 & 2 & 3 & 3 & 2 & 2 & \\
\hline 3 months & 3 & 4 & 3 & & & 5 & & & 3 & 6 & & & \\
\hline$>12$ months old & & & & & & & & & & & & & 4 \\
\hline
\end{tabular}

(2012) recently demonstrated that recombinant $\alpha$-synuclein and A $\beta$ fibrils are wholly sufficient to transmit disease, thereby suggesting a common pathogenic mechanism of disease progression for neurodegenerative brain amyloidoses. Although Clavaguera et al. (2009) showed that diseased mouse brain lysates containing pathological tau induce spread of tau pathology in recipient mice and Liu et al. (2012) and de Calignon et al. (2012) demonstrated trans-synaptic spreading of tau pathology in mouse brains, the nature of tau species that drive interneuronal transmission are unknown, and it is unclear whether tau fibrils alone are sufficient to transmit tau pathology. Here we show that injection of preformed fibrils (pffs), assembled from recombinant human tau protein into the brains of young human mutant tau Tg mice (PS19) before the known onset of tauopathy, induced widespread transmission of $\mathrm{AD}$-like tau pathology.

\section{Materials and Methods}

Tau Tg mice. PS19 tau Tg mice were generated using a cDNA encoding the human 1N4R tau isoform with the P301S tau gene (MAPT) mutation that is pathogenic for familial FTLD-Tau driven by the murine prion protein promoter (Yoshiyama et al., 2007). The strain was maintained on a B6C3 background.

Generation of tau pffs from recombinant tau. We cloned cDNAs encoding the longest human isoform of tau (2N4R) with a myc tag at the $3^{\prime}$ end with the P301S MAPT mutation (T40/PS) in addition to a truncated form of human tau (K18/PL) containing only the four MT-binding repeats with a myc tag at the $5^{\prime}$ end and the P301L MAPT mutation into NdeI/EcoRI sites of pRK172 bacterial expression vectors. Purified tau was induced to fibrillize in the presence of heparin, and successful fibrillization was confirmed using a Thioflavin (Th) T fluorescence assay and electron microscopy as described previously (Guo and Lee, 2011). Aliquots of tau pffs were stored at $-80^{\circ} \mathrm{C}$ until use when they were sonicated with 40 brief pulses before injection into the brains of PS19 mice. Truncated $\alpha$-synuclein fibrils (1-120) were made as described previously (Luk et al., 2012) and used as a control.

Stereotaxic surgery. All experiments were performed in accordance with protocols approved by the Institutional Animal Care and Use Committee of the University of Pennsylvania.

After deeply anesthetizing 2- to 3-month-old PS19 mice of either sex with a ketamine/xylazine mixture, they were immobilized in a stereotaxic frame (David Kopf Instruments), and stereotaxic injections were made using predetermined coordinates (see Figs. $1 A, 3 A$ ) with a Hamilton syringe under aseptic conditions. All injected animals were observed during and after surgery, and an analgesic was administered after surgery. The 2- to 3-month-old PS19 mice were separately injected into hippocampus (bregma, $-2.5 \mathrm{~mm}$; lateral, $+2 \mathrm{~mm}$; and depth, $-1.8 \mathrm{~mm}$ ) or striatum (bregma, $+0.2 \mathrm{~mm}$; lateral, $+2 \mathrm{~mm}$; and depth, $-2.6 \mathrm{~mm}$ ) and overlaying cortex (bregma, $+0.2 \mathrm{~mm}$; lateral, $+2 \mathrm{~mm}$; and depth, -0.8 $\mathrm{mm}$ ) with the following: (1) T40/PS recombinant tau pffs ( $2 \mu \mathrm{g} / \mu \mathrm{l}) ;(2)$ K18/PL recombinant tau pffs $(2 \mu \mathrm{g} / \mu \mathrm{l})$; (3) PBS vehicle in a final volume of $2.5 \mu \mathrm{l}$; or (4) human $\alpha$-synuclein (1-120) pffs $(2 \mu \mathrm{g} / \mu \mathrm{l})$. The total volume injected per site was $2.5 \mu \mathrm{l}$ for all mice. The number of mice used for each experimental condition is summarized in Table 1.

Histology and immunohistochemistry. At predetermined postinjection time intervals, all injected mice were deeply anesthetized, transcardially perfused with $30 \mathrm{ml}$ of PBS, followed by $10 \%$ neutral buffered Formalin (NBF), after which the brains and spinal cords were removed, postfixed overnight by immersion in NBF, and then thoroughly rinsed in PBS, followed by embedding in paraffin blocks from which $6-\mu \mathrm{m}$-thick sections were cut through the entire CNS (brain, brainstem, spinal cord) for conventional histochemical staining and immunohistochemistry (IHC). CNS sections were stained using a polymer horseradish peroxidase detection system (Biogenex) and counterstained with hematoxylin. For detection of tau pathology, we immunostained every 20 slides with the monoclonal antibodies (mAbs) AT8 (specific for tau phosphorylated at Ser202/Thr205, 1:10,000; Innogenetics) and MC1 (specific for a pathological conformation of tau, 1:2000; a generous gift from Peter Davies, Feinstein Institute for Medical Research, Manhasset, NY) for all mice used in this study. Other antibodies used are as follows: mAb T49 (specific for mouse tau, 1:1000; Yoshiyama et al., 2007), affinity-purified acetylated Lys280 (ac-K280) (specific for acetylated tau, 1:2000 with citrate buffer treatment; Cohen et al., 2011), mAb TG3 (specific for a conformation-dependent phosphorylated tau epitope, 1:250; a generous gift from Peter Davies), polyclonal antibody 17025 (specific for total tau, 1:1000; Yoshiyama et al., 2007), and NeuN (specific for neurons, 1:500; Millipore). Sections were also stained with ThS to detect tau tangles that exhibited histological properties of amyloid and by hematoxylin and eosin to visualize CNS architecture. Because the relative resistance of inclusions to proteinase $\mathrm{K}(\mathrm{PK})$ digestion is another known feature of amyloids, we assessed tau pathology for this property by treating selected deparaffinized sections with $10 \mu \mathrm{g} / \mathrm{ml}$ PK for $1 \mathrm{~min}$, followed by IHC.

For quantitative analysis of MC1-positive neurons within and around the locus ceruleus (LC), we selected slides from -5.0 to $-6.0 \mathrm{~mm}$ bregma at $\sim 30 \mu \mathrm{m}$ intervals to stain with MC1 (1:2000) and counted MC1-positive neurons. To detect noradrenergic LC neurons, we stained slides with anti-tyrosine hydroxylase (TH) antibody. Quantification of neurons with tau tangles was expressed as MC1-positive neurons as a percentage of total number of TH-positive LC neurons.

The area occupied by MC1-positive neurons in each mouse at the injection sites in the hippocampus and in the contralateral hippocampus was assessed using $4 \times$ images, and caudal regions of the lateral entorhinal cortex of both hemispheres (approximately $-4 \mathrm{~mm}$ bregma) were assessed using $20 \times$ images without counterstain. Quantification was performed by NIH ImageJ software (Carroll et al., 2011; Zhang et al., 2012). NeuN-positive cell counts in the medial CA3 region of each mouse and the area occupied by MC1-positive neurons with and without PK treatment in lateral CA3 and LC were quantified using $20 \times$ images without counterstain by NIH ImageJ as well (Zhang et al., 2012). For semiquantitative analysis of MC1-positive neurons in the entire CNS, we selected six different coronal CNS levels (bregma, 0.98, - 1.22, -2.18 , $-2.92,-4.48$, and $-5.52 \mathrm{~mm}$ for hippocampus injections; and bregma, $1.98,0.38,-2.18,-2.92,-4.48$, and $-5.52 \mathrm{~mm}$ for striatum and overlaying cortex injections), marked brain regions according to anatomical/ 
cytoarchitectural patterns (Paxinos and Franklin, 2003), and used a previously published grading system adapted from the Braak staging system for AD to score the extent of tau pathology in pff-injected PS19 mice (Hurtado et al., 2010; Zhang et al., 2012). After grading individual brain regions in each mouse, we imported averaged values of these scored into CNS heat maps using custom-designed heat-map software to create pathology distribution maps for the telencephalon and brainstem.

Western blot analyses. PBS-perfused unfixed brains were used for biochemical analysis by dissecting the hippocampi and neocortices separately. Before analysis, the brain samples were sonicated in $4 \mathrm{vol} / \mathrm{g}$ RIPA buffer ( $50 \mathrm{~mm}$ Tris, $150 \mathrm{~nm} \mathrm{NaCl}, 0.1 \%$ SDS, $0.5 \%$ sodium deoxycholate, $1 \%$ NP-40, 5 mм EDTA, 1 mм PMSF, $0.1 \%$ protease inhibitor mixture, and $0.5 \%$ phosphatase inhibitor, $\mathrm{pH} 8.0$ ) and centrifuged at $100,000 \times g$ for $30 \mathrm{~min}$ at $4^{\circ} \mathrm{C}$. The supernatants were saved as RIPA-soluble fractions, whereas the RIPA-insoluble pellets were washed with $1 \mathrm{~m}$ sucrose in RIPA buffer to remove myelin and associated lipids and centrifuged at $100,000 \times g$ for $30 \mathrm{~min}$ at $4^{\circ} \mathrm{C}$. The RIPA-insoluble pellets were then extracted in $1 \mathrm{vol} / \mathrm{g}$ tissue with $2 \%$ SDS buffer (50 mm Tris-HCl, $\mathrm{pH} 7.6$ ). Soluble and insoluble fractions were analyzed by SDS-PAGE, followed by Western blotting using anti-tau antibody, paired helical filament 1 (PHF1) to detect tau phosphorylated at Ser396/404. We loaded $10 \mu \mathrm{g}$ of total proteins for RIPA fractions and 100 and $20 \mu \mathrm{g}$ of total proteins for SDS fractions from cortical and hippocampal extractions, respectively.

Statistical analyses. Quantification results were analyzed using one-way ANOVA, followed by Tukey's post hoc test or Student's $t$ test in GraphPad Prism 4.0 software (GraphPad Software). Data were expressed as mean \pm SEM, and differences with $p<0.05$ were considered significant.

\section{Results}

\section{Induction and spreading of tau pathologies after brain} injections of synthetic tau pffs

To test whether synthetic tau pffs assembled from recombinant full-length human tau with the P301S mutation (T40/PS pffs) can induce tau pathology in young PS19 mice (2-3 month old), we performed unilateral injection of T40/PS pffs into the hippocampus (Fig. $1 A$ ) and examined brain tau pathology at 1,3 , and 6 months after injection. Because the PS19 Tg mice normally do not develop tau pathology until 8-9 months of age, PBS injected age-matched control PS19 mice (Fig. $1 B$ ) failed to show any pathology at 3 months after injection $(n=3)$ when stained by MC1, as was the case for mice injected with synthetic truncated human $\alpha$-synuclein (1-120) fibrils $(n=4)$ (Fig. $1 C)$, suggesting that tissue damage from injections of PBS or inoculation with unrelated pffs do not nonspecifically induce tau pathology. However, 1 month after tau pff injection, numerous neurons near the injection site, including CA1 and CA3 in the ipsilateral hippocampus, showed prominent tau inclusions in perikarya and neurites that were intensely labeled by MC1 (Fig. 1D). Moreover, tau pathology spread to parts of hippocampus rostral (data not shown) as well as caudal to the injection site and also to the caudal ipsilateral entorhinal cortex (Fig. 1D, Caudal Ent Ctx). Interestingly, pathological tau accumulations were found in the contralateral hippocampus as well, but mainly in the CA3 region. Because the uninjected PS19, PBS-injected PS19, and $\alpha$-synuclein pff-injected PS19 mice never show detectable tau accumulations at this age (Fig. $1 B, C$ ) and because all the observed tau pathologies were seen exclusively in mice injected with tau pffs, this is consistent with the view that injected tau pffs act as "seeds" to recruit endogenous overexpressed tau to form intracellular tau aggregates.

The extent of tau pathology in the ipsilateral hippocampus rapidly plateaued such that no obvious increase in the pathology was observed at 3-6 months after T40/PS pff injection compared with 1 month after injection. However, tau pathology continued to spread and increase over time in the contralateral hippocam- pus and caudal entorhinal cortex (Fig. 1D), as well as other CNS regions. We also performed hippocampal injections using K18/PL pffs containing only the MT-binding repeats with the P301L mutation (Guo and Lee, 2011) and found a similar pattern of spreading of tau pathology, indicating that this fragment of tau is sufficient to recruit and convert tau into aggregates and drive transmission of tau pathology (Fig. 1E).

\section{Dose- and time-dependent transmission of tau pff induced tauopathy}

To determine whether lower doses of tau pffs are capable of inducing tau pathology, we compared injections of $0.05,0.5$, or 5 $\mu \mathrm{g}$ of K18/PL or T40/PS pffs into hippocampus and found that even the lowest dose of pffs $(0.05 \mu \mathrm{g})$ induced tau pathology recognized by $\mathrm{MC} 1$ in a few dentate granule neurons close to the injection site as early as 2 weeks after injection (Fig. 2A). A doseresponse relationship was observed at 2 weeks and 1 month after injection because a dose of $0.5 \mu \mathrm{g}$ induced a larger number of MC1-positive neurons, which increased further with the highest dose injected $(5 \mu \mathrm{g})$. Concomitant with this dose-dependent increase in the number of MC1-positive neuronal perikarya, we observed extensive MC1-positive neuritic tau pathology with the highest dose of pffs at 2 weeks and 1 month after injection. Remarkably, distinct MC1 immunoreactivity was seen immediately around injection sites at 2 weeks after injection of 0.5 or $5 \mu \mathrm{g}$ pffs, and this became more abundant at 2 weeks to 1 month after injection. A similar pattern was observed with AT8 staining (Fig. $2 A$, right). These results suggest that tau pffs can rapidly convert endogenous tau into hyperphosphorylated pathological conformers, and the effects are dose and time dependent.

Quantitative analysis revealed that percentage of area occupied by MC1-positive inclusions significantly increased over time in both injected and contralateral hippocampus until 3 months after injection, followed by a reduction at 6 months (Fig. $2 B, C$ ). In contrast, pathology in the ipsilateral caudal entorhinal cortex remained unchanged from 3 to 6 months (Fig. 2D), whereas pathology in the contralateral entorhinal cortex continued to increase after 3 months (Fig. 2E). To investigate whether a decrease of $\mathrm{MC1}$-positive neurons in hippocampus at 6 months after injection was associated with neuronal loss, we quantified number of neurons in the medial CA3 regions of each mouse using $\mathrm{NeuN}$ antibody, but found no significant change in neuron numbers over the entire postinjection period (Fig. $2 F$ ).

\section{Region-specific spreading of pff-induced pathologies}

To assess the extent of tau pathology spread after injections of tau pffs into other brain regions, we examined the brains of animals 1,3 , and 9 months after K18/PL pff injection into striatum and overlaying neocortex (Fig. $3 A$ ) and observed strong MC1 immunoreactivity within 1 month of injection, as well as at 3 and 9 months after injection (Fig. 3B). Notably, pathological tau was transmitted to many brain regions that were distinct from those affected by hippocampal injection (Fig. 3B), including the substantia nigra and thalamus, which are interconnected with the striatum (Fig. 3B). At 9 months after pff injection, we also detected remarkable MC1 immunoreactivity along white matter tracts in corpus callosum. Thus, injection of tau pffs into different brain regions resulted in widespread transmission of tau pathology to diverse brain regions that are anatomically interconnected with the injection sites. Interestingly, although significant numbers of tangle-like accumulations were detected in the overlaying neocortex, almost no tau pathology was detected in striatum (Fig. 3B). Similar to hippocampal injections, dose- and 
A
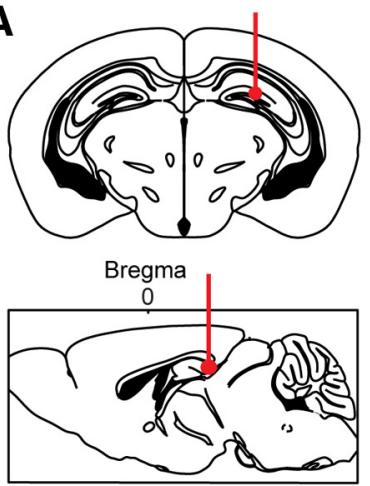

D
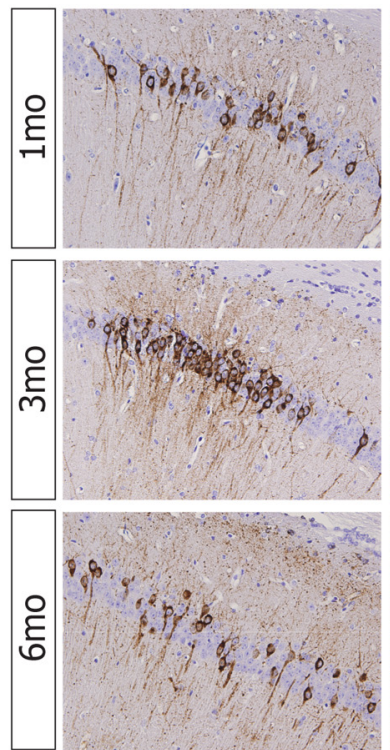

E
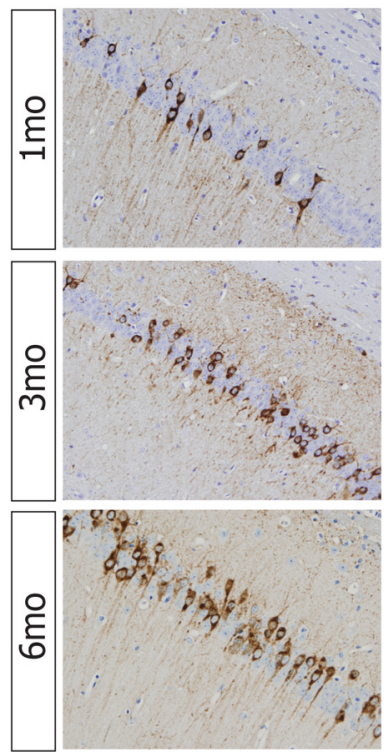

B

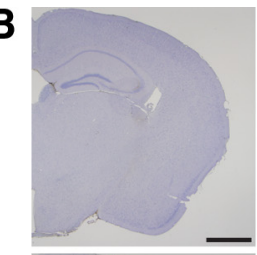

C
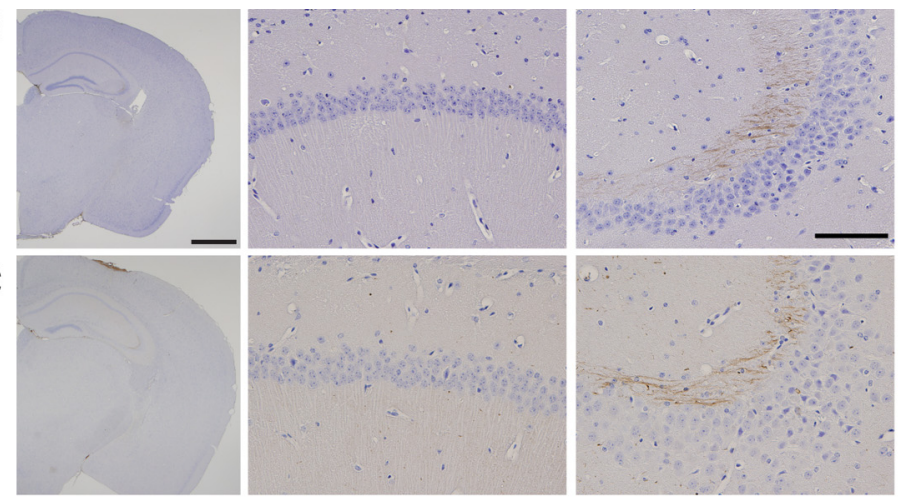

Lateral CA3
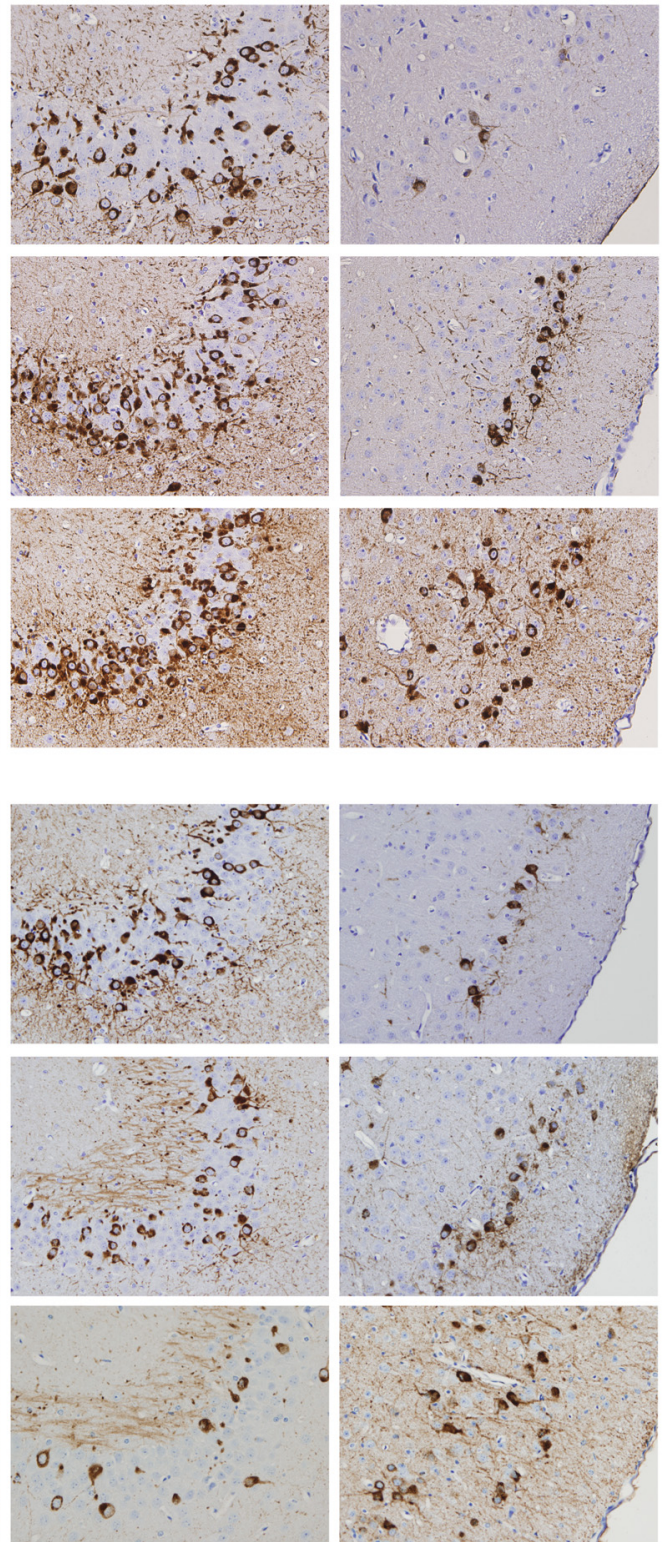

Contralateral CA3

T40/PS fibrils
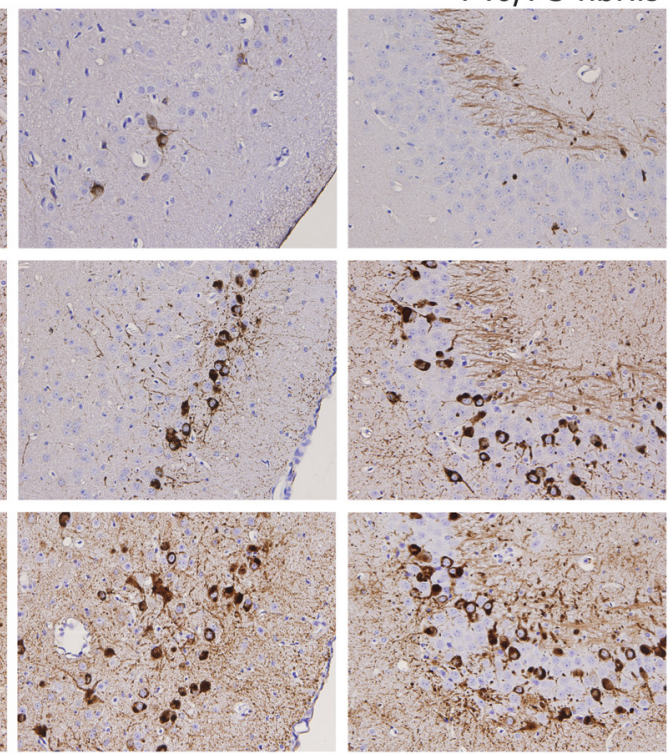

K18/PL fibrils
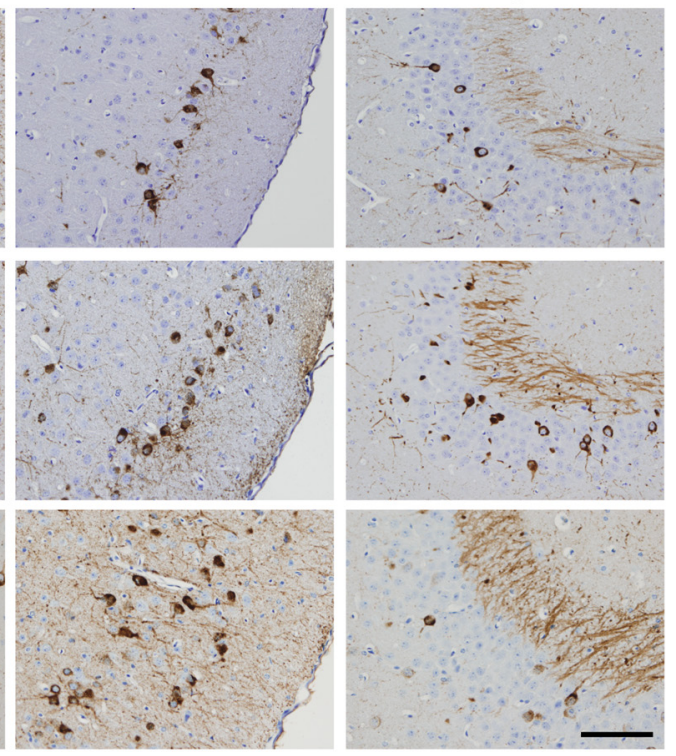

Figure 1. Injection of T40/PS and K18/PL pffs into hippocampus induces tauopathy in young PS19 Tg mice. A, Schematic illustration of injection site. Top and bottom show coronal and sagittal planes, respectively. Red line indicates injection path and red dot indicates injection site. $\boldsymbol{B}$ and $\boldsymbol{C}$ are microscopic images of mouse brains 3 months after injection with PBS and synthetic human $\alpha$-synuclein pffs, respectively, immunostained with mAb MC1. The three columns display low-power images of injected hemispheres, high-power images of CA1 region, and lateral CA3 region of injected hippocampus, in this order. Scale bars: left column, $1 \mathrm{~mm}$; right column, $100 \mu \mathrm{m}$. D, Microscopic images of brain sections immunostained with MC1 showing, from left to right, the ipsilateral hemisphere of CA1 and lateral CA3, as well as the caudal part of entorhinal cortex (Caudal Ent (Xt) and the contralateral CA3, from PS19 mice injected with T40/PS fibrils survived for 1 month ( $n=3$ ), 3 months $(n=3)$, and 6 months $(n=2)$ after injections. $\boldsymbol{E}$, Same as $\boldsymbol{D}$ except that K18/PL pffs were inoculated into the hippocampus of PS19 mice. $n=5$ for each survival period. Scale bar, $100 \mu \mathrm{m}$. 
A
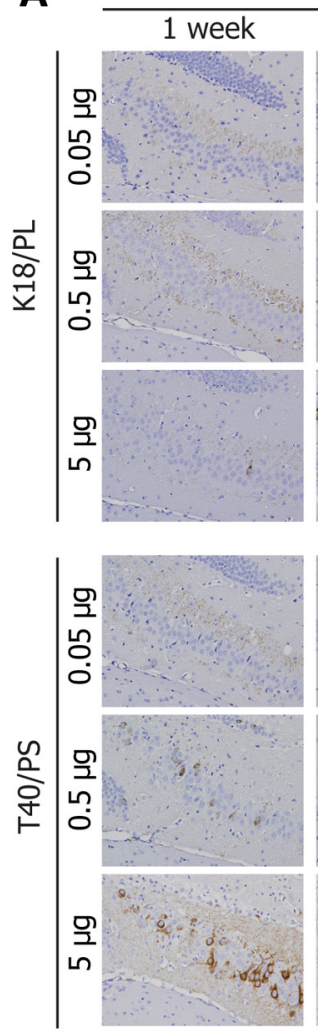

B

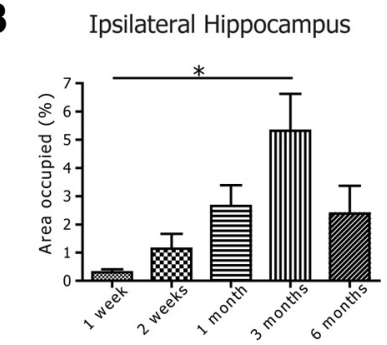

C

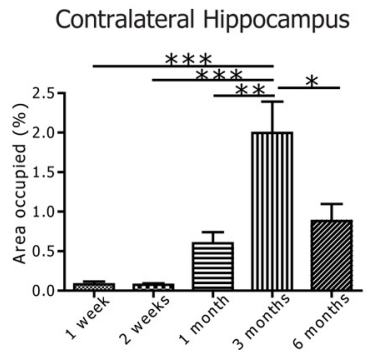

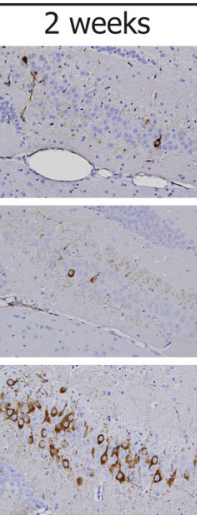
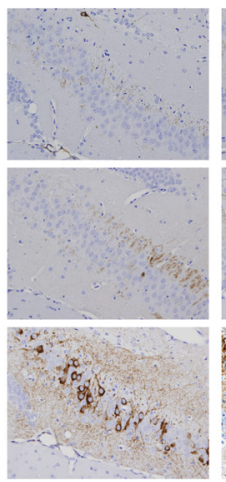

D

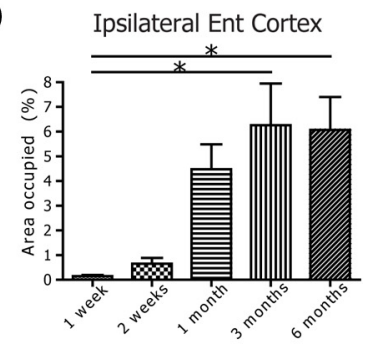

$\mathbf{E}$

Contralateral Ent Cortex

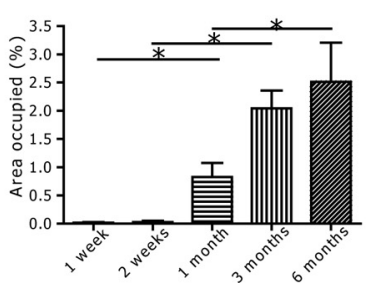

AT8
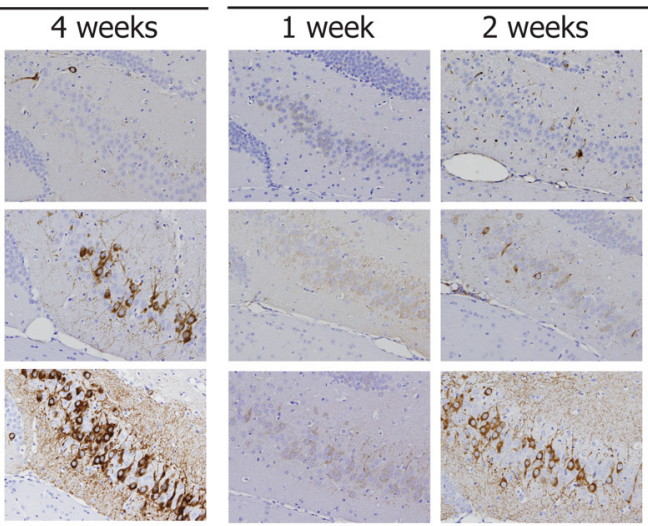

4 weeks
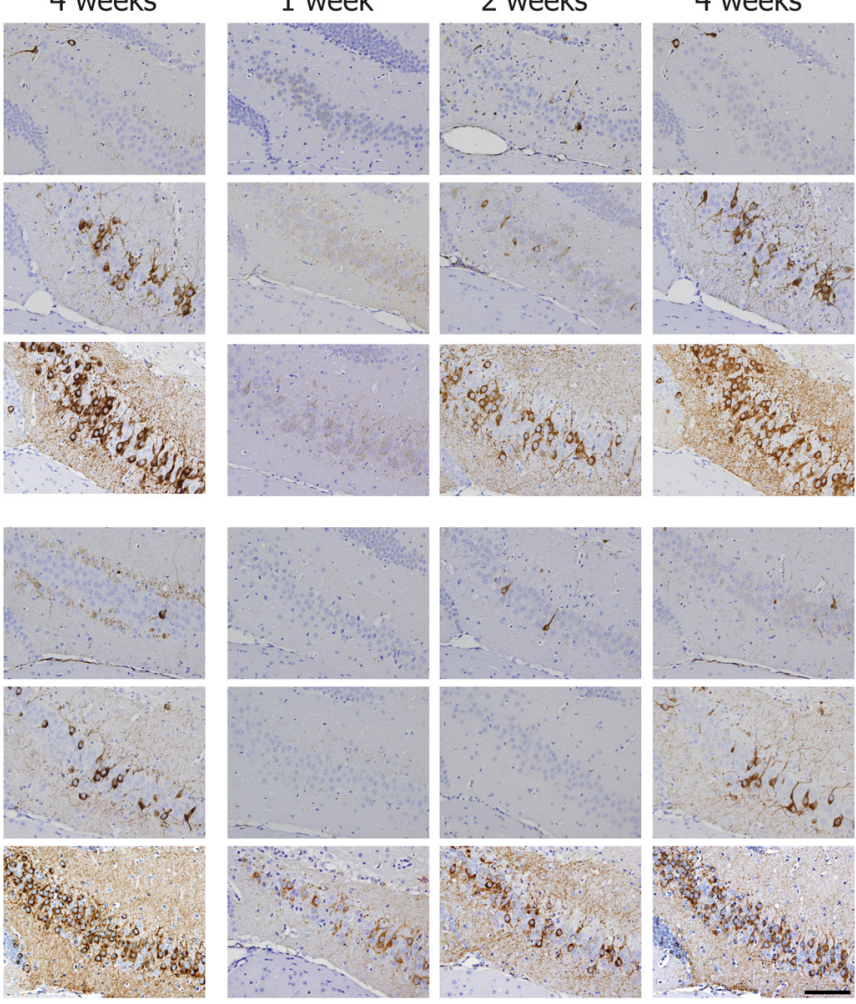

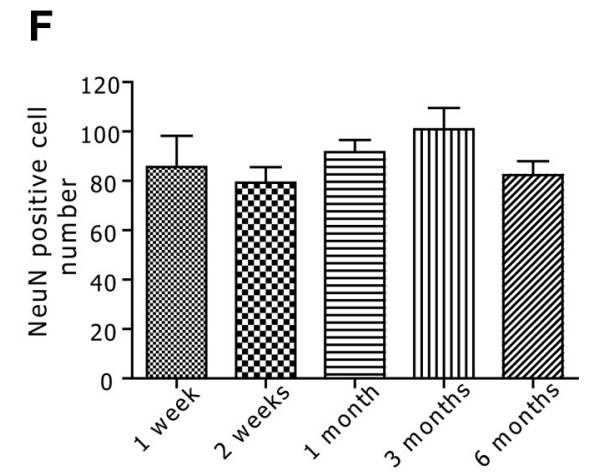

Figure 2. Effects of tau pff injections into hippocampus are dose and time dependent. $A$, Different amounts of K18/PL pffs $(0.05,0.5$, and $5 \mu \mathrm{g})$ (top) or T40/PS pffs ( $0.05,0.5$, and $5 \mu \mathrm{g})$ (bottom) were injected into hippocampus, and mice were killed at 1,2, and 4 weeks after injection. Each panel shows medial part of CA3 and dentate gyrus. Brain sections were stained with MC1 (left) and AT8 (right). Scale bar, $100 \mu \mathrm{m} . \boldsymbol{B}-\boldsymbol{E}$, Quantitative analyses of MC1-positive neurons in ipsilateral hippocampus ( $\boldsymbol{B}$ ), contralateral hippocampus (C), ipsilateral entorhinal cortex (D), and contralateral entorhinal cortex (E). Animals used for quantitative analyses were combined from both T40/PS and K18/PL injections. $n=5$ for 1 week, $n=4$ for 2 weeks, $n=8$ for 1 month, $n=8$ for 3 months, and $n=7$ for 6 months. Significant differences between pairwise comparisons were marked with stars. ${ }^{*} p<0.05,{ }^{* *} p<0.01,{ }^{* * *} p<0.001$. $F$, Quantitative analysis of NeuN-positive neurons at different time points after injection. There is no significant difference between any postinjection periods. The number of animals used for this analysis was the same as $\boldsymbol{B}-\boldsymbol{E}$. Ent $\mathrm{Cortex}$, Entorhinal cortex.

time-dependent effects of K18/PL pffs were seen after injection into striatum and overlaying neocortex, although the highest dose of $5 \mu \mathrm{g}$ resulted in pathology that quickly plateaued at 1 week after injection (Fig. 3C).

Intriguingly, survey of the entire brain for tau pathology in pff-injected PS19 mice revealed that LC neurons consistently displayed significant tau pathology, especially on the ipsilateral side within a short period of time after injection, independent of injection sites (i.e., hippocampus or striatum/neocortex) and type of tau pffs injected (i.e., full-length T40/PS or truncated K18/PL pffs) (Fig. $4 A, B$ ), which is consistent with the widespread projections of LC neurons. As shown by lower-power microscopic images, $\mathrm{MC1}$-positive neurons were found exclusively in LC among all pontine structures (Fig. $4 A, B$, first column). Double labeling 
A

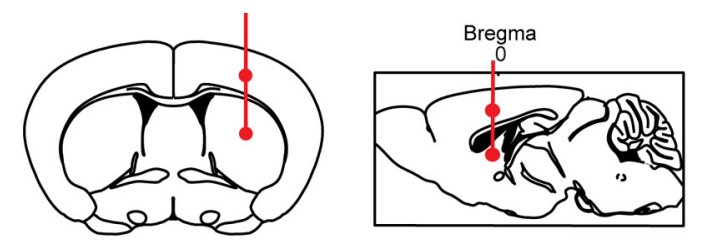

B
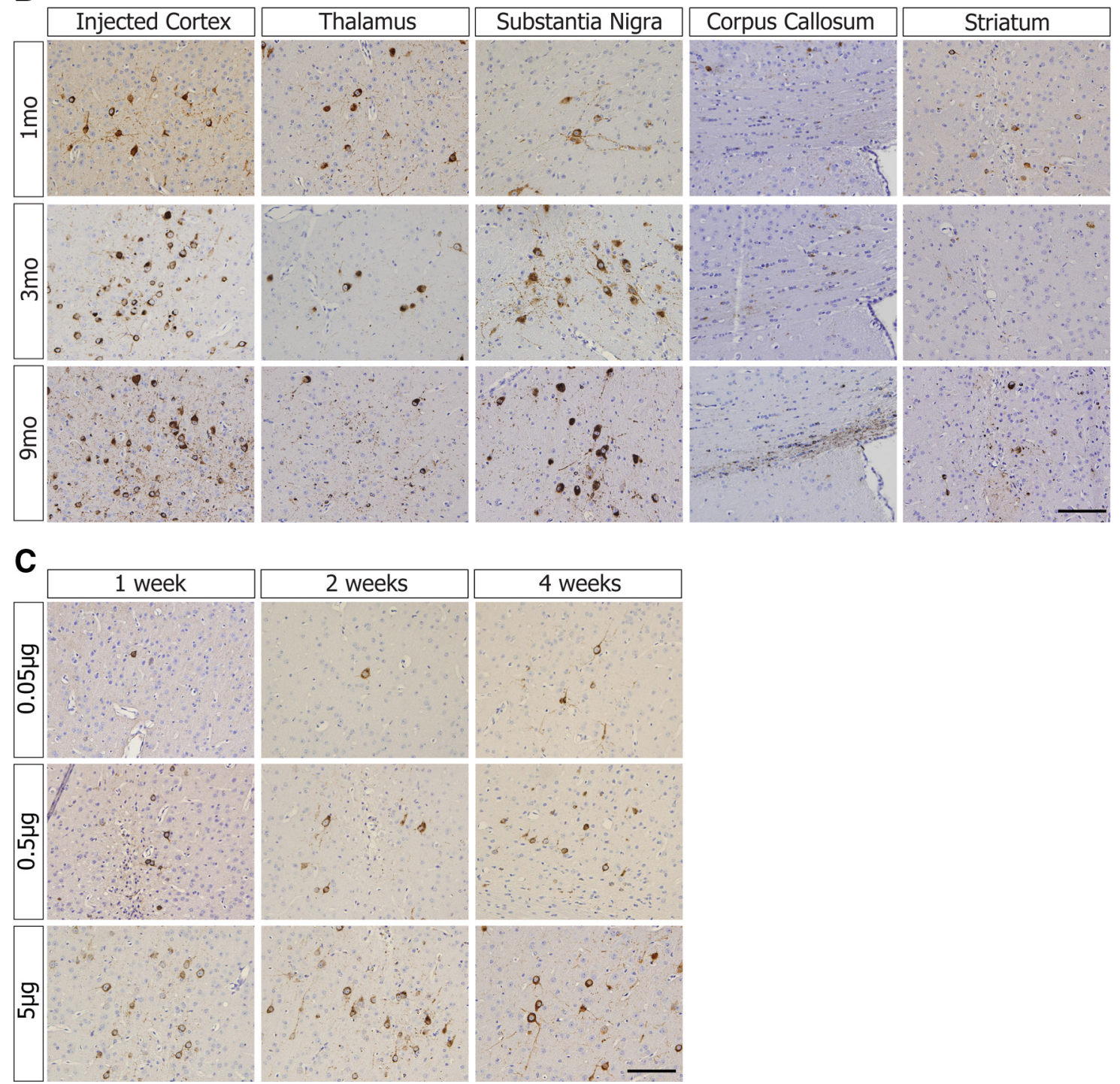

Figure 3. Injection of K18/PL pffs into striatum and overlaying cortex induces tauopathy. $A$, Schematic illustrations of injection sites with coronal and sagittal planes. Red line indicates injection path, and red dot indicates injection site. $\boldsymbol{B}$, Microscopic images of ipsilateral cortex, thalamus, substantia nigra, corpus callosum, and ipsilateral striatum (from left to right) from PS19 mice immunostained with MC1 after injection with K18/PL pffs and survived for $1(n=3), 3(n=6)$, and $9(n=3)$ months. C, Different amounts of K18/PL pffs $(0.05,0.5$, and $5 \mu \mathrm{g})$ were injected into striatum and overlaying cortex, and mice were killed at 1 week, 2 weeks, and 1 month after injection. Each panel shows injected cortex. Two mice were used for each concentration of tau pffs injection and each survival period. Brain sections were stained with MC1. Scale bars, $100 \mu \mathrm{m}$.

using MC1 and an anti-TH antibody confirmed the presence of pathological tau in a subset of TH-expressing LC neurons (Fig. $4 C$ ). Quantitative analysis of MC1-positive neurons along the rostral-caudal axis of LC revealed a time-dependent increase of pathology with the different injection paradigms, which reached maximum when $\sim 8-10 \%$ of TH-positive neurons developed tangle-like inclusions (Fig. 4D).

To compare the transmission patterns of tau pathologies induced by pff injections into hippocampus versus striatum and overlaying neocortex, we conducted a CNS-wide survey to assess the regional abundance and distribution of tau inclusions at different times after injection and summarized semi- quantitative analysis in the form of heat maps as shown in Figures 5 and 6. As expected, hippocampal injection resulted in bilateral distribution of tau pathology spreading throughout the hippocampus and interconnected regions, including the caudal entorhinal cortex, amygdala, piriform cortices, and LC in a time-dependent manner (Fig. 5). Similarly, pff injections into striatum and overlaying neocortex also led to the transmission of tau inclusions to regions interconnected with these injection sites, including thalamus, substantia nigra, dorsal raphe nuclei (probably because of the striatal injection), layer IV of neocortex, and LC (probably because of the neocortical injection) (Fig. 6). 
A

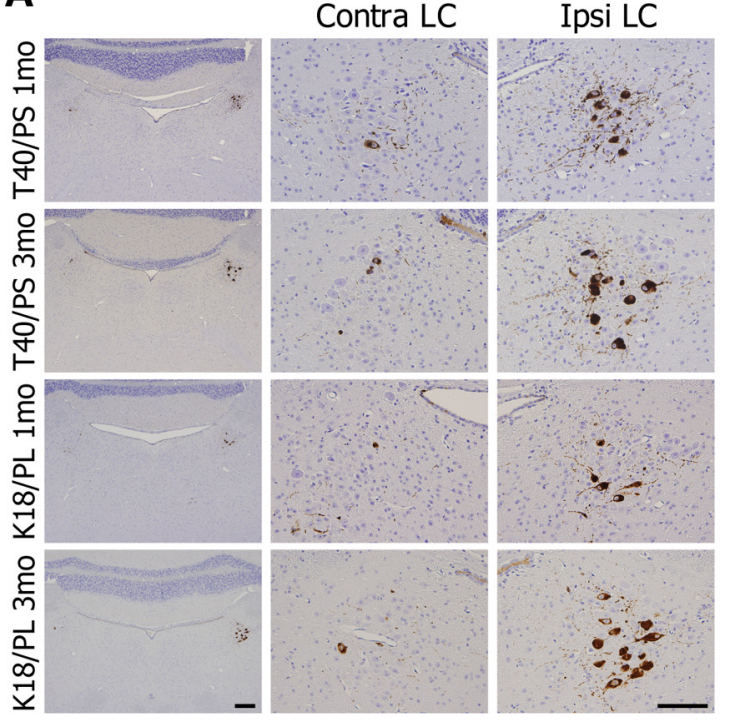

B

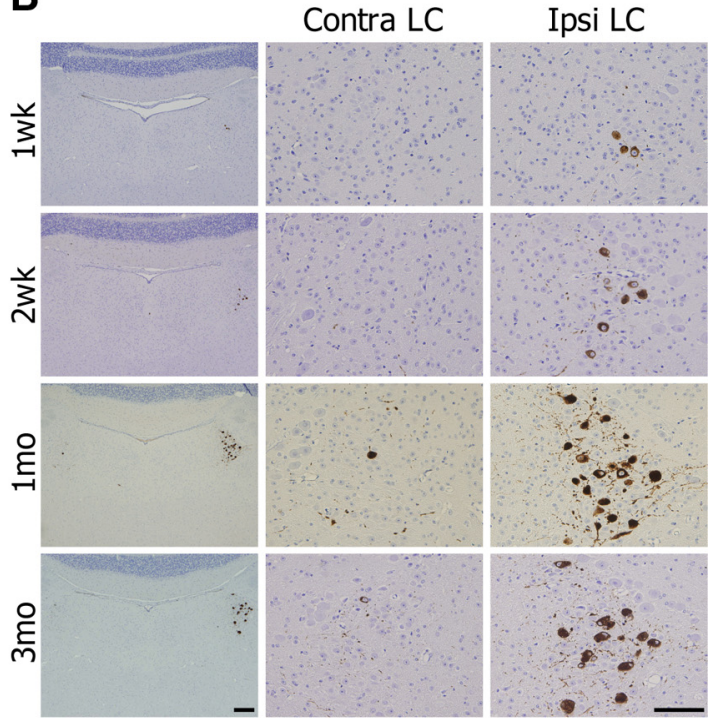

c
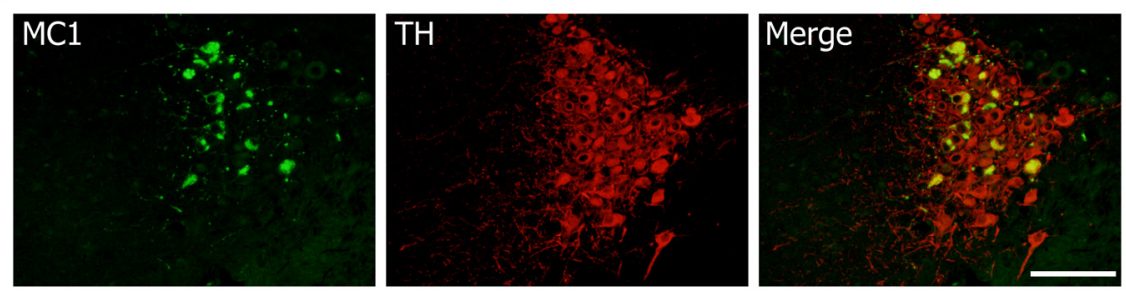

D

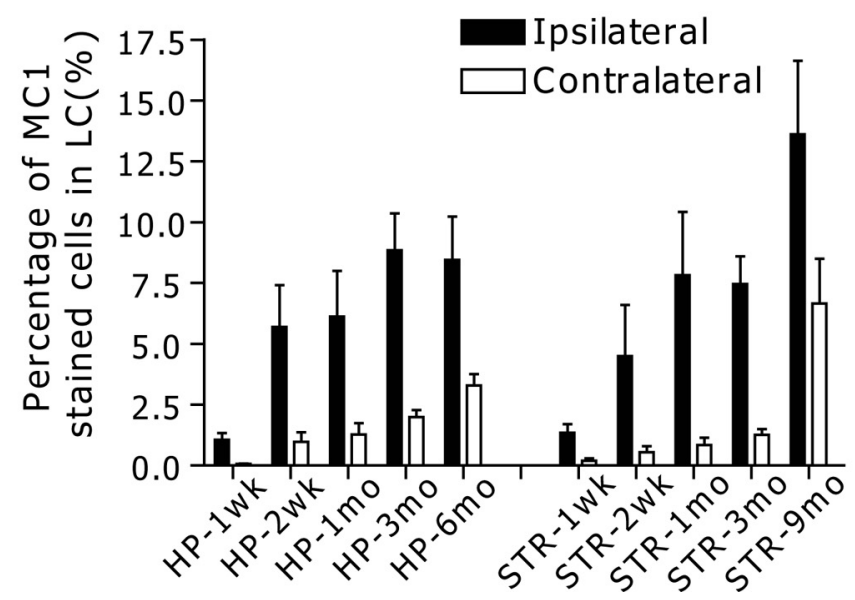

Figure 4. Injections of tau pffs transmit tau pathology to the LC. A, T40/PS pffs (top 2 panels) or K18/PL pffs (bottom 2 panels) injection into hippocampus. Lower-power microscopic images of brainstem (left), medial region of LC of contralateral (middle) and ipsilateral (right) to injection site, at 1 month (top) and 3 months (bottom) after pff inoculation are shown stained with MC1. Scale bars: left, $200 \mu \mathrm{m}$; right, $100 \mu \mathrm{m} . \boldsymbol{B}, \mathrm{K} 18 / \mathrm{PL}$ injection into striatum and overlaying cortex at 1 week (wk), 2 weeks, 1 month (mo), and 3 months after the injection. Scale bars: left, $200 \mu \mathrm{m}$; right, $100 \mu \mathrm{m}$. C, MC1 (green) and TH (red) in two-color immunofluorescence staining, which revealed that many MC1-positive neurons are colocalized with TH cells in LC (merge). Scale bar, $100 \mu \mathrm{m} . \mathbf{D}$, Quantitative analysis of MC1-positive LC neurons. $n=2-9$ mice for each group. Black bars represent ipsilateral, and white bars represent contralateral.

\section{Synthetic tau pffs induce tau pathologies that closely resemble $A D$ NFTs}

To further characterize the nature of pff-induced tau pathologies, we performed IHC using a panel of different anti-tau antibodies in injected PS19 mice survived for different lengths of time. We also compared pff-induced tangle-like inclusions in injected mice with 14-month-old symptomatic uninjected PS19 mice that have abundant tau pathology at this age. These studies showed the following.

(1) Using a mouse-specific tau MAb (T49), we found that endogenous mouse tau also became aggregated as early as 2 weeks after injection, whereas uninjected animals even at 14 month of age showed hardly any mouse tau pathology (Fig. 7A). Double staining with T49 and 17025 (total tau polyclonal antibody) confirmed that endogenous mouse tau was indeed recruited into pathological tau inclusion (Fig. 7B).

(2) The pff-induced tau lesions were not only detected by mAbs AT8 and MC1 (Fig. 1D,E) but also MAb TG3 (specific for phosphorylation at Thr231/Ser235 and conformation dependent), whereas TG3 detected little to no pathology in aged uninjected mice (Fig. 7A) that did manifest tau pathology detected by other tau antibodies. 
Hippocampus injection

1 month

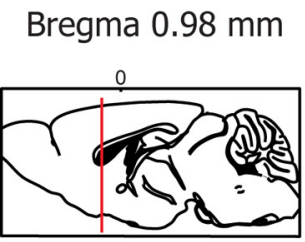

Bregma - $1.22 \mathrm{~mm}$

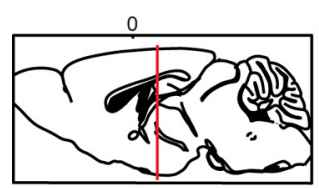

Bregma $-2.18 \mathrm{~mm}$

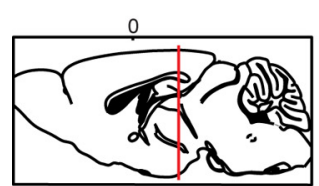

Bregma - $2.92 \mathrm{~mm}$

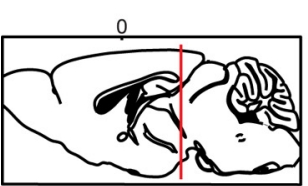

Bregma $-4.48 \mathrm{~mm}$

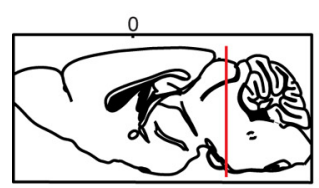

Bregma $-5.52 \mathrm{~mm}$

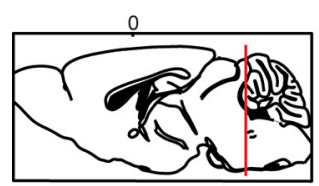

3 months
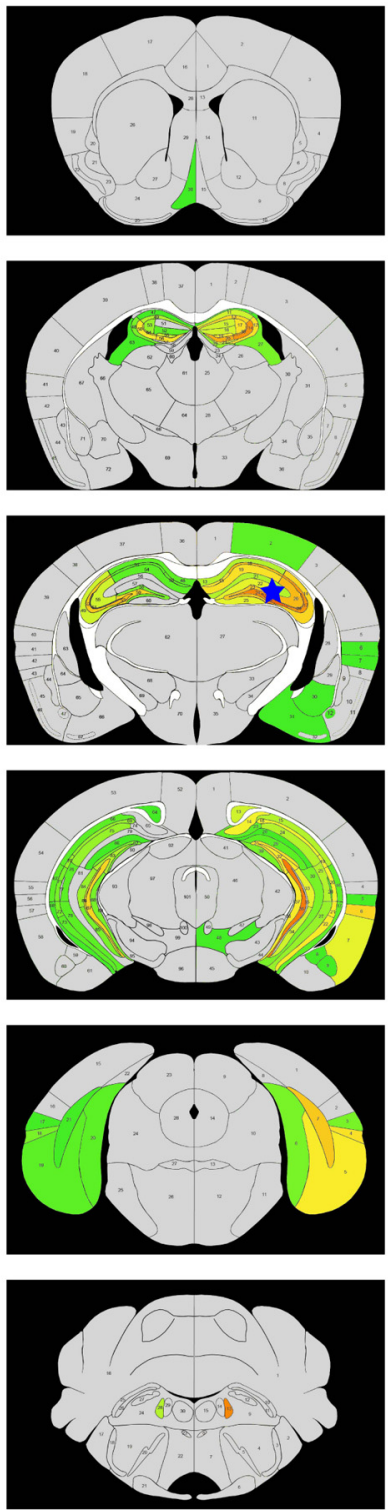
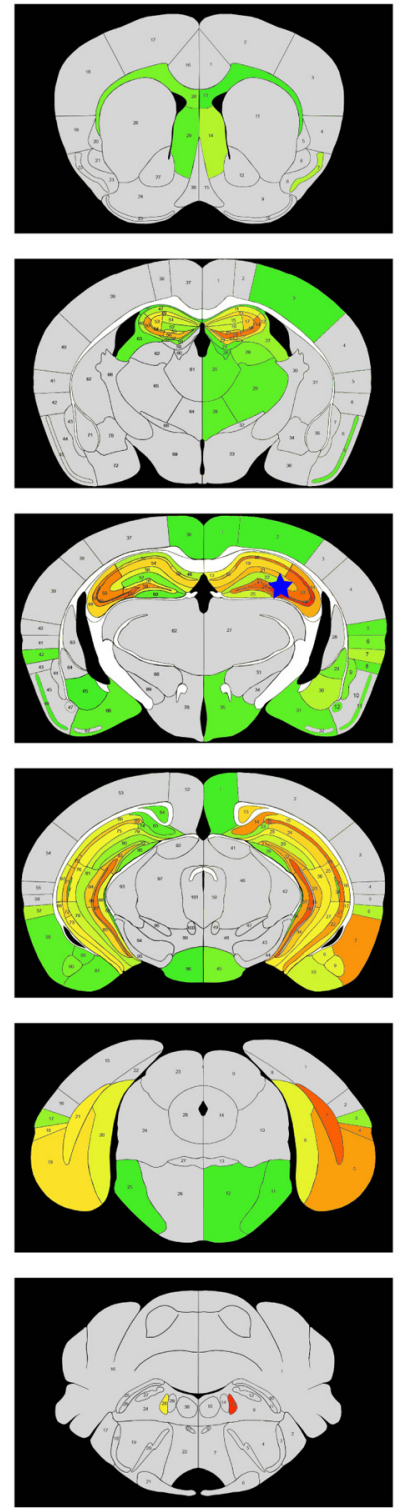

6 months
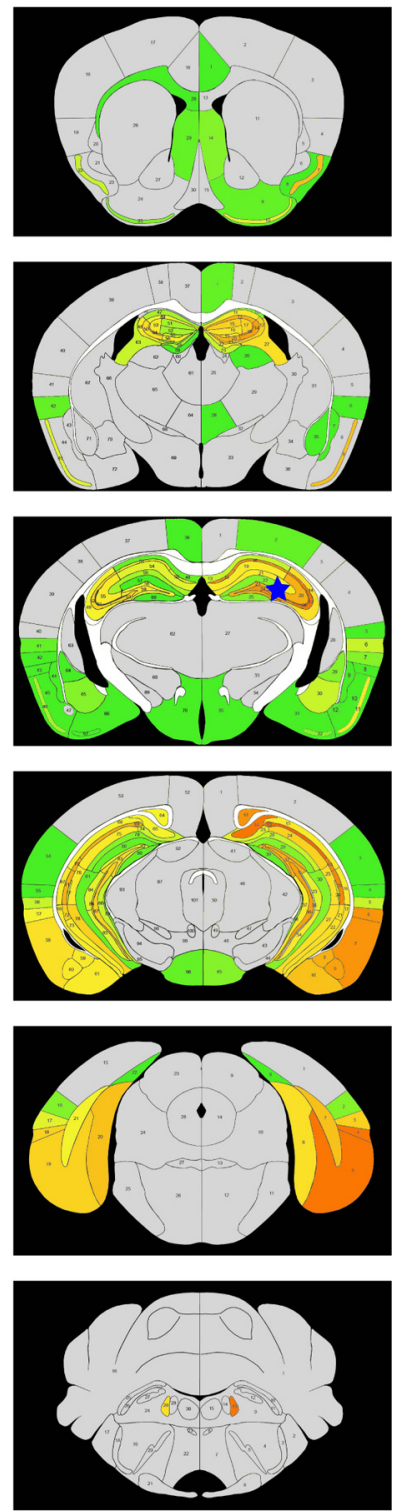

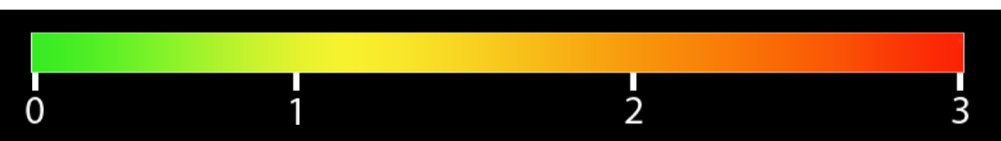

Figure 5. Heat maps of tau pathology in coronal sections stained with MC1 for hippocampus injection. Semiquantitative analyses of tau pathology graded using MC1 antibody. Each panel represents heat map pathology distribution on one of the six different coronal planes (bregma, $-0.98,-1.22,-2.18,-2.92,-4.48$, and $-5.52 \mathrm{~mm})$ at $1 \mathrm{month}(n=8), 3 \mathrm{months}(n=8)$, or 6 months ( $n=7)$ after tau pff injection into hippocampus. Left column shows sagittal view of the selected coronal planes used to generate the heat maps. Blue star indicates injection site.

(3) Using a polyclonal antibody specific for ac-K280 tau, we found that pff-induced tau tangles were acetylated similar to human AD and other tauopathy brains (Cohen et al., 2011; Irwin et al., 2012), whereas acetylated tau is hardly detected in uninjected aged symptomatic PS19 mice. Double staining using MC1 and ac-K280 demonstrated that most pathological inclusions contain acetylated tau (Fig. 7B).

(4) Finally, pff-induced tau inclusions became ThS positive starting 2 weeks after injection and the intensity and abundance of ThS staining increased over time, whereas tau pathologies in uninjected PS19 mice never showed appreciable ThS staining.
Because immunostaining by TG3 and ac-K280 and ThS positivity in $\mathrm{AD}$ and other human tauopathies are signatures of more mature NFTs, tau pff-induced tau tangles more closely resemble authentic AD-like human NFTs. Moreover, mature NFTs (i.e., those showing anti-ac-K280 immunostaining and ThS positivity) are present in all the different affected brain regions, including medial CA3, caudal cortex, and LC of pff-injected (Fig. $8 A$ ) but not uninjected aged PS19 mice (Fig. $8 B$ ) and double labeling with $\mathrm{MC} 1$ and ThS revealed that most pff-induced tau inclusions show properties of amyloid as indicated by ThS positivity beginning at $\sim 1$ month after injection (Fig. $8 C$ ). 


\section{Striatum and cortex injection}

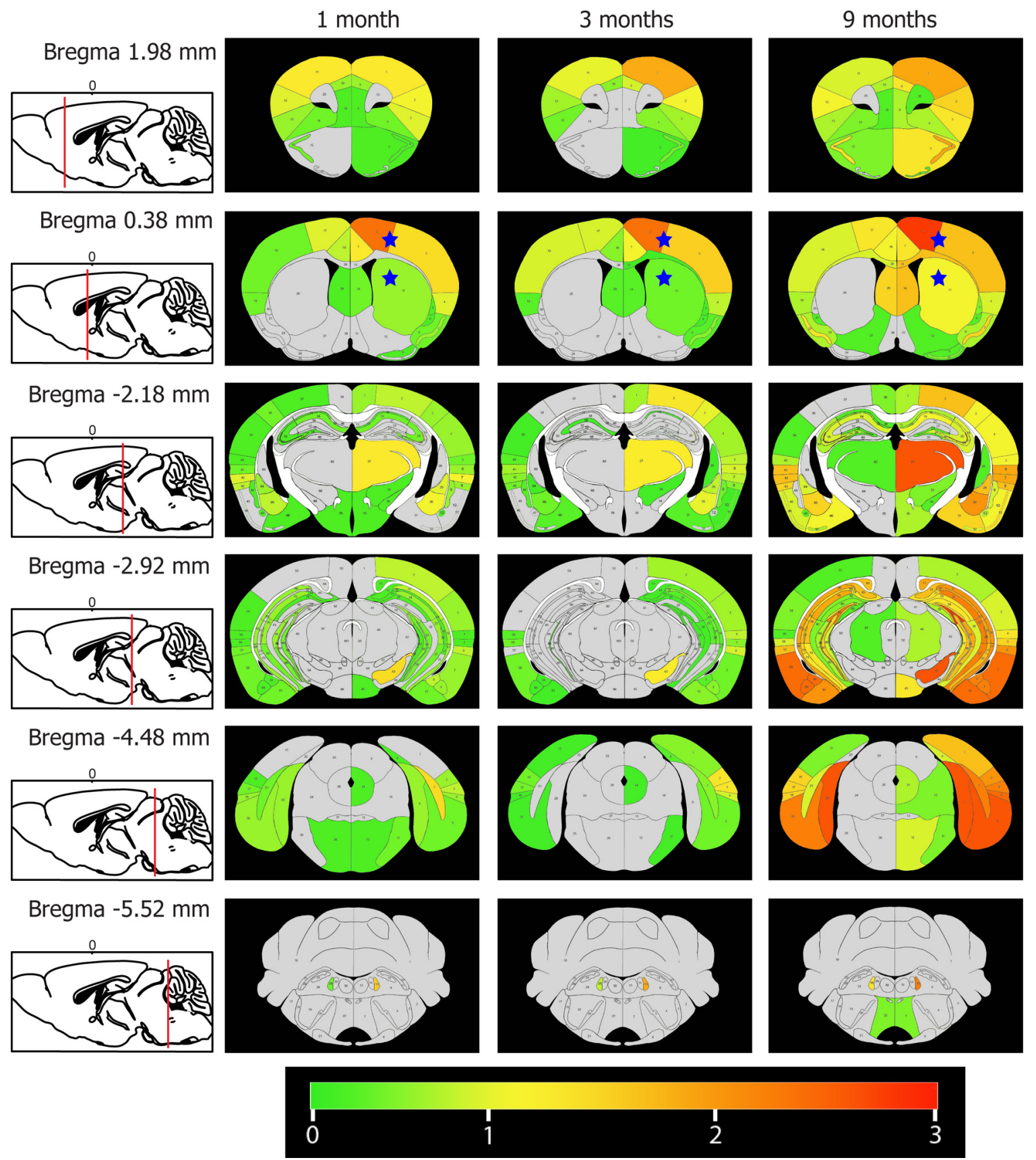

Figure 6. Heat maps of tau pathology in coronal sections stained with MC1 for striatum and overlaying cortex injection. Semiquantitative analyses of tau pathology graded using MC1 antibody. Each panel represents heat-map pathology distribution on one of the six different coronal planes (bregma, 1.98, 0.38, $-2.18,-2.92,-4.48$, and $-5.52 \mathrm{~mm}$ ) after 1 month $(n=6), 3$ months $(n=9)$, and 9 months $(n=5)$ injection into striatum and overlaying cortex. Left column shows sagittal view of the selected coronal planes. Blue stars indicate injection sites.

To further distinguish tau pathologies developed in pffinjected mice versus those in older uninjected PS19 Tg mice, we treated brain slides with PK before immunostaining with MC1. Although PK treatment reduced MC1 immunoreactivity in both groups of mice, pathology in pff-injected mice was significantly more resistant to PK treatment than that in uninjected aged PS19 $\mathrm{Tg}$ mice, suggesting that pff-templated tau tangles contain more $\beta$-pleated sheet amyloid structures (Fig. 9A,B). Finally, immunoblot analysis confirmed that, at 3 months after injection into hippocampus, lysates from both cortex and hippocampus of the 6-month-old pff-injected mice contained significantly more in- soluble hyperphosphorylated tau when compared with agematched uninjected PS19 mice (Fig. 9C).

\section{Discussion}

Our study demonstrated that intracerebral injection of synthetic tau pffs into the brains of young PS19 mice induced rapid formation of NFT-like tau inclusions at injection sites that was followed by a time- and dose-dependent transmission of tau pathology to more distant synaptically connected brain areas. The induction and transmission of tau pathology did not occur in age-matched control PS19 mice injected with PBS or $\alpha$-synuclein pffs in the 

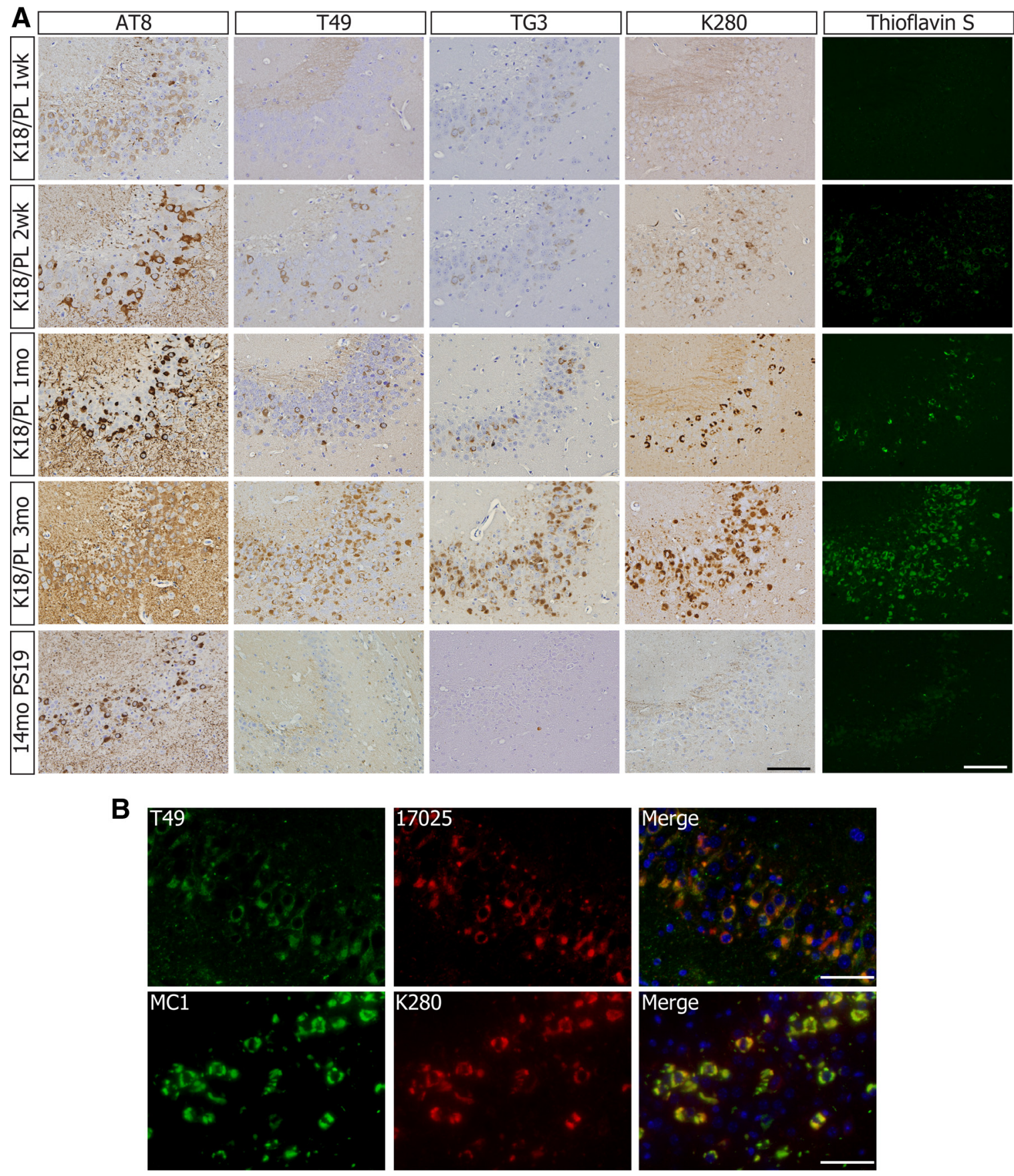

Figure 7. Properties of tau tangles in tau pff-injected versus uninjected aged PS19 mice. $A$, Brain sections were immunostained with mAbs AT8 to detect hyperphosphorylated tau, T49 to detect mouse tau, TG3 to detect specific conformation and phosphorylation changes, anti-ac-K280 rabbit antibody to detect acetylation at K280, and ThS histochemistry to detect mature NFT-like structures after the injection of K18/PL pffs into hippocampus at 1 week, 2 weeks, 1 month, and 3 months after injection. Comparison was made with a 14-month-old uninjected PS19 mouse. Scale bars, 100 $\mu \mathrm{m} . \boldsymbol{B}$, Double immunofluorescence with T49 (green) and 17025 (red) (top) or MC1 (green) and K280 (red) (bottom). Scale bars, $50 \mu \mathrm{m}$.

same brain regions, eliminating nonspecific effects of injection itself or nonspecific responses to inundation with aggregated proteins. Significantly, synthetic pffs assembled from both fulllength and truncated recombinant human tau proteins transmitted tau pathology, thereby supporting the notion that synthetic tau fibrils alone are sufficient to induce endogenous tau to acquire NFT-like misfolding near inoculation sites and propagate to widespread interconnected brain regions via neural networks.

Previous in vitro studies established that synthetic tau pffs are capable of templating the conversion of soluble tau into fibrillar aggregates in cultured cells (Frost et al., 2009; Guo and Lee, 2011;
Kfoury et al., 2012). Furthermore, one in vivo study showed that inoculation of brain lysates containing abundant pathological tau from diseased mutant tau-expressing mice can induce tau pathology in wild-type tau-expressing mice that otherwise do not form tau inclusions (Clavaguera et al., 2009). Although this study also showed that immunodepletion of tau from these lysates eliminated their ability to induce tau pathology, the identity and nature of the transmissible tau species in the injected brain lysates were not resolved, nor could it be determined whether other non-tau components in the lysates had contributed to the transmission of tau pathology. More recently, Lasagna-Reeves et al. 
(2012) demonstrated propagation of tau pathology in wild-type mice after injection of tau oligomers isolated from $\mathrm{AD}$ brain, but the purity of tau species immunoprecipitated from brain homogenates is questionable. Hence, through the use of synthetic fibrils made from pure recombinant protein, our study provides the first direct and compelling evidence that fibrillar species of tau alone are entirely sufficient to recruit and convert endogenous soluble tau into pathological aggregates in neurons or neuronal processes, followed by transmission of tau pathology to other brain regions from a single injection site in vivo.

The route of spreading seems to coincide with synaptic connections between the injection site and affected brain regions, accounting for different patterns of tau pathology transmission that resulted from pff injections into hippocampus or striatum/overlaying neocortex. Moreover, injection into hippocampus or striatum/overlaying cortex consistently induced tau pathology in the LC, a brain structure far away from injection sites. LC, the noradrenergic nucleus in the brainstem, innervates nearly the entire brain except the basal ganglia (Foote et al., 1983; for review, see Berridge and Waterhouse, 2003). With its widespread efferent projections, the LC-norepinephrine (LC$\mathrm{NE}$ ) system is involved in the regulation of not only arousal state but also various cortical functions (for review, see AstonJones and Cohen, 2005). Although aged PS19 mice develop tau pathology in the LC, it occurred only when animals show widespread tau aggregates, neuron loss, and motoric phenotypes (Yoshiyama et al., 2007; our unpublished data). In contrast, pff-injected PS19 mice displayed LC pathology within a short postinjection period (1-2 weeks), regardless of whether inoculation occurred in hippocampus or cortex. Because LC receives afferent input from highly limited brain regions, namely the paragigantocellularis and the nucleus prepositus hypoglossus (Aston-Jones et al., 1986), LC tau inclusions observed here most likely reflect retrograde transmission from these injection sites that receive synaptic inputs from LC.

In stark contrast to LC, direct pff injection into the striatum failed to develop tau pathology at the injection site even 3 months after injection, demonstrating differential vulnerability of distinct brain areas to tau aggregation. The LC-NE system has been implicated in neurodegenerative diseases, including AD and Parkinson's disease (Chan-Palay, 1991; German et al., 1992; for review, see Marien et al., 2004). Recent studies even suggest that earliest AD NFTs may actually

B
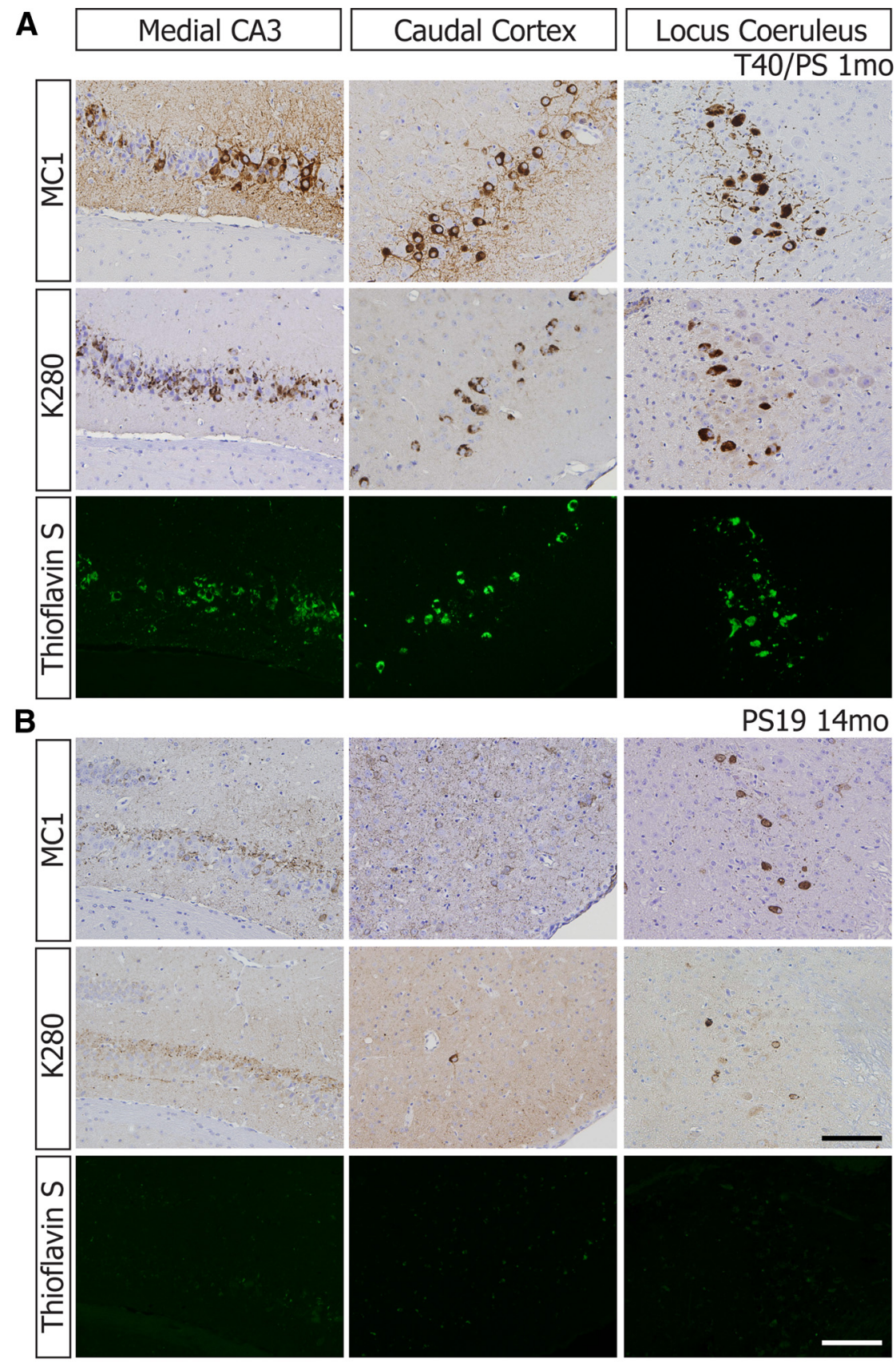

PS19 14mo
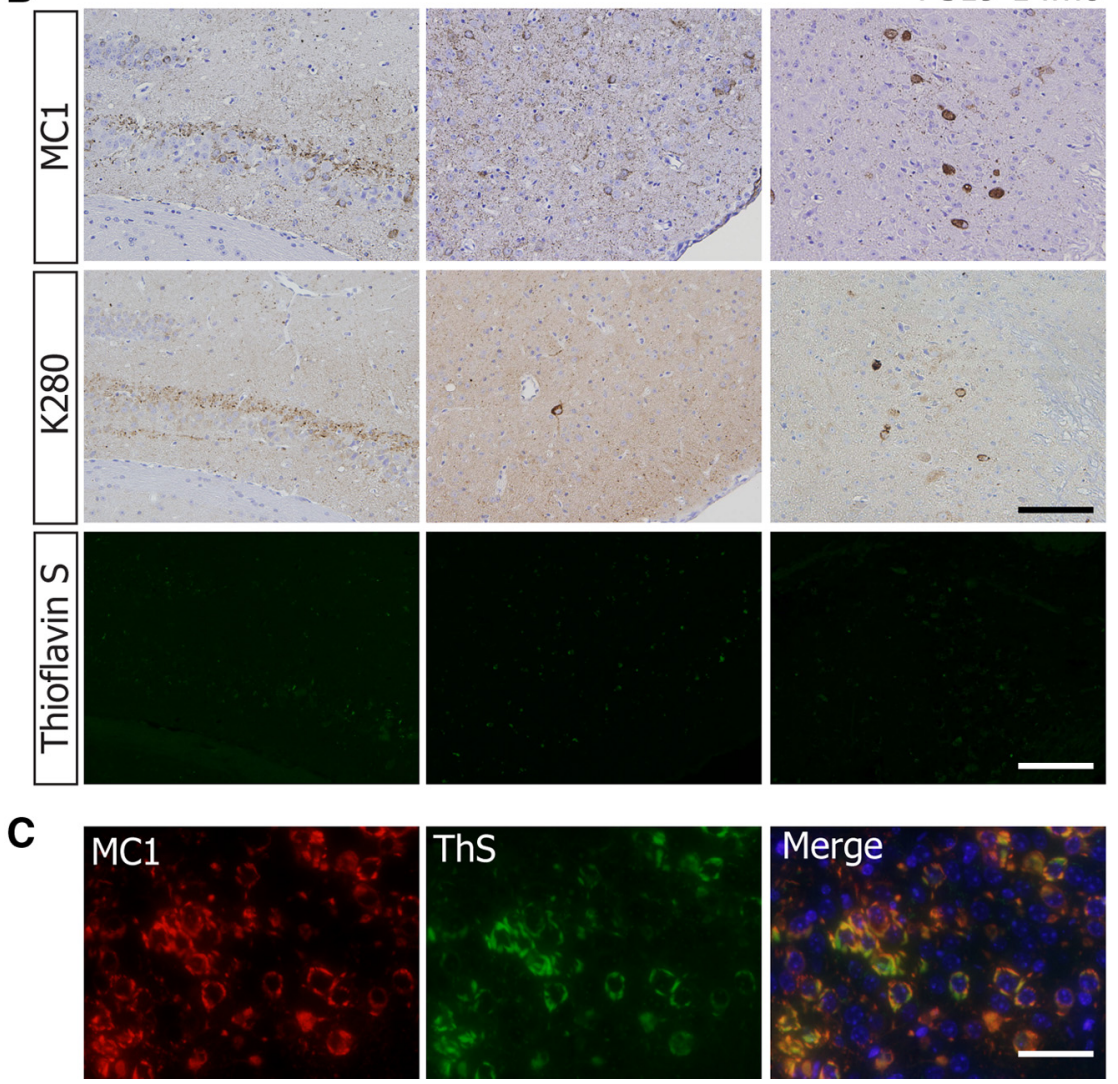

Figure 8. Comparison between T40/PS pff-injected and normally aged PS19 mice in different brain regions. Brain sections were immunostained with MC1, anti-ac-K280 antibody, and ThS. Three different brain regions are shown, i.e., medial part of CA3 in hippocampus, caudal part of the cortex, and LC of T40/PS pff-injected PS19 (A) and uninjected aged PS19 (B). Scale bars, $100 \mu \mathrm{m}$. C, Double immunofluorescence with MC1 (red) and ThS (green). Scale bar, $50 \mu \mathrm{m}$.

initiate in a subset of subcortical nuclei, including LC (Braak and Del Tredici, 2011a, 2011b). This is consistent with the selective vulnerability of LC in developing tau pathology in our study. Together with its widespread synaptic projections, LC may play critical roles in the progression of tau pathology in AD. Addi- 
A

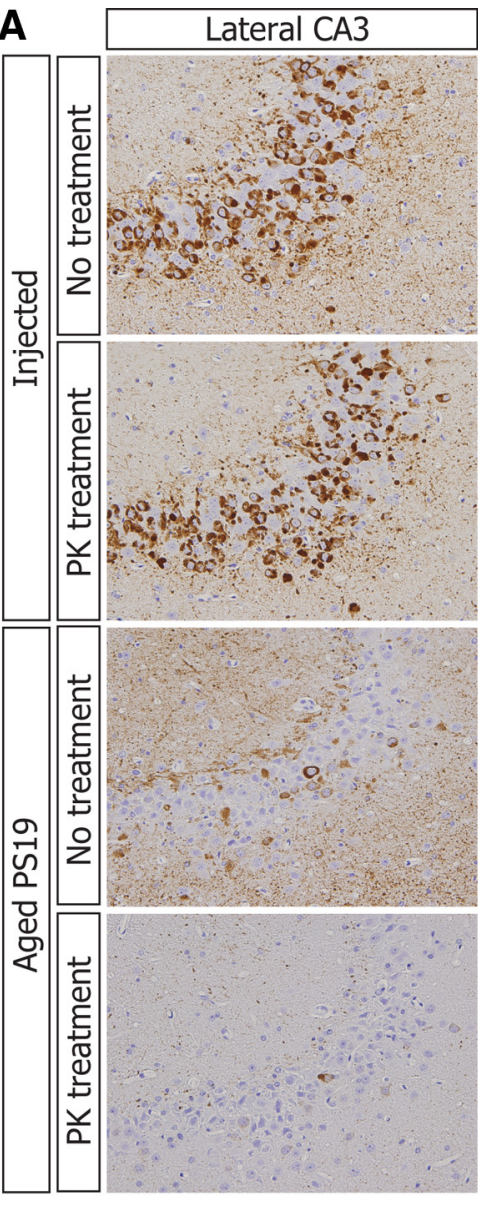

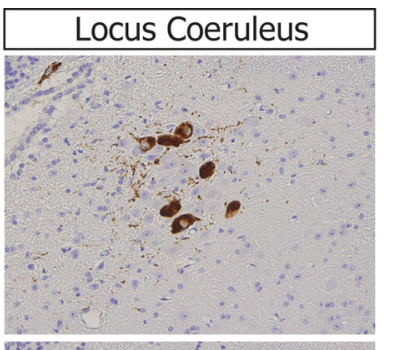
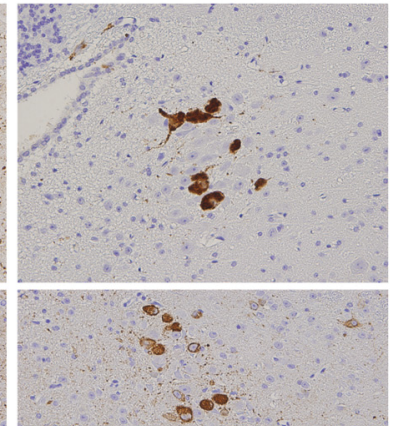

8

- : : 9

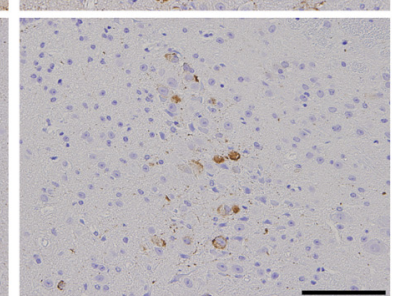

C

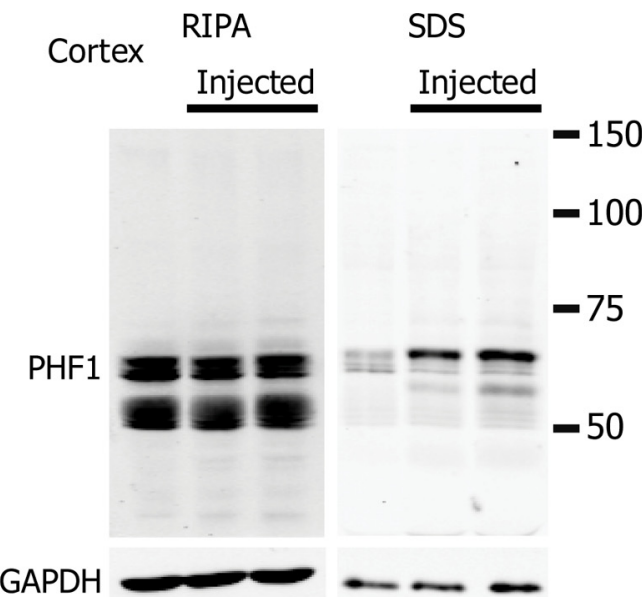

$\rightarrow$

B

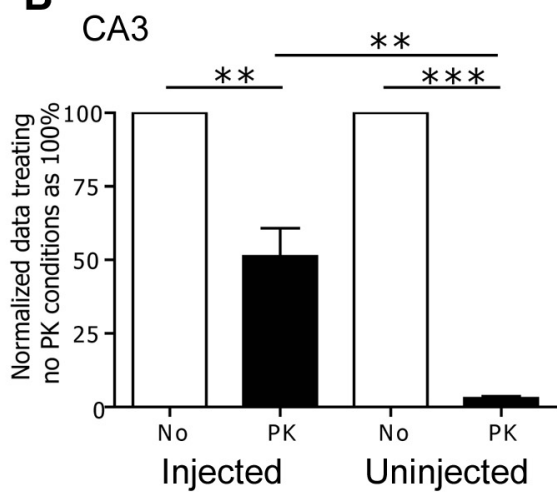

LC

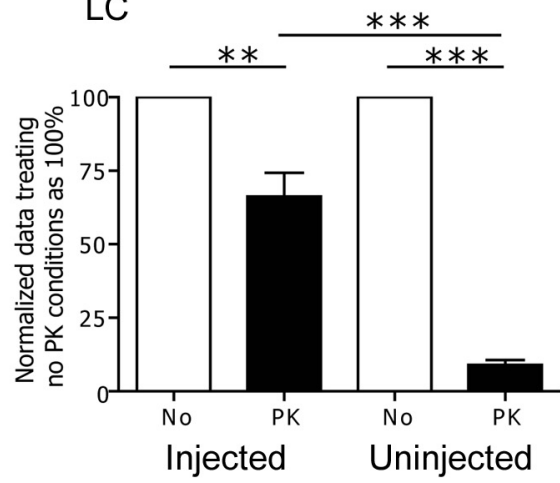

RIPA SDS

Hippocampus
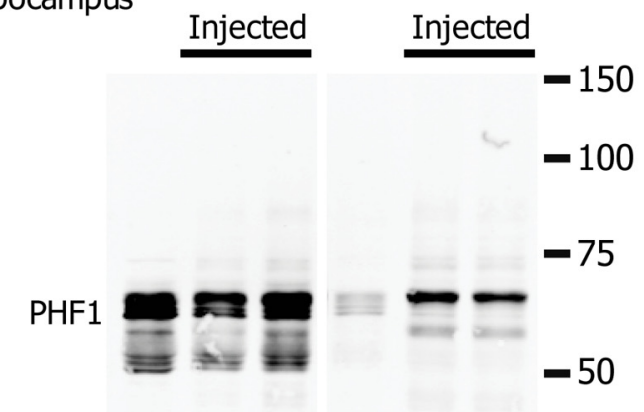

GAPDH

Figure 9. Comparison of the effects of PK treatment on pff-injected and uninjected aged PS19 mice. $A$, MC1 stained hippocampus (lateral CA3) and LC of K18/PL pff-injected mouse (top panels) and uninjected aged PS19 mouse (bottom panels) with no treatment or with $10 \mu \mathrm{g} / \mathrm{ml} \mathrm{PK} \mathrm{treatment.} \mathrm{Scale} \mathrm{bar,} 100 \mu \mathrm{m}$. B, Quantitative analyses of MC1-positive neurons in CA3 and LC. Data represents MC1 immunoreactivity normalized to no PK treatment conditions for both injected $(n=5)$ and uninjected aged $(n=4)$ PS19 mice. White bars represent no treatment, and black bars represent PK treatment. ${ }^{* *} p<0.01,{ }^{* * *} p<0.001$. C, Immunoblot analysis of cortex and hippocampus extracted sequentially with RIPA buffer, followed by $2 \%$ SDS. On each blot, the leftmost lane is uninjected age-matched control, and the other two lanes are two different injected mice (Injected) at 3 months after injection. mAb PHF1 detects hyperphosphorylated tau, and GAPDH was used as a loading control.

tional studies are required to elucidate the relationship between LC-NE system dysfunction and etiology of tauopathy.

The PS19 mice used in this study were first reported in 2007, but over time we observed attenuated formation of tau pathology (Yoshiyama et al., 2007). For example, although hyperphosphorylated tau inclusions were prominent at 6 month of age in our previous study, similar pathology only emerges after the mice are
$>12$ month of age. Also, significant neuronal death that initially began in 9-month-old PS19 mice now occurs after 12 months of age (Zhang et al., 2012). Thus, we injected tau pffs into 2- to 3 -month-old PS19 mice to ensure that endogenous tau pathology would not be present, and this was confirmed in our PBS-injected age-matched and uninjected control PS19 mice even at the latest time point of the study (i.e., 6 months after injection). Hence, we 
can confidently attribute the formation and spread of tau pathology in the pff-injected mice to the recruitment of endogenous tau by injected tau pffs.

Interestingly, although the onset and severity of tau pathology in our current PS19 mice is highly variable, injection of tau pffs resulted in a more uniform onset and distribution of tau pathology. Importantly, we observed that tau aggregates developed after pff injection are qualitatively different from that in aged PS19 mice because endogenous mouse tau is readily recruited into tau aggregates in pff-injected mice but not uninjected aged PS19 mice. Also, pff-seeded tau inclusions share key features with mature NFTs in AD brains in that they are intensely immunostained by TG3, a conformation-specific phospho-tau mAb, by our antiac-K280 tau antibody and display strong ThS binding, properties that are not shared by tau inclusions in uninjected aged PS19 mice. Finally, pff-seeded NFT-like structures are more resistant to PK digestion than non-seeded tau aggregates. Together, our data support that the pathogenesis of pff-seeded tau tangles is mechanistically different from tau accumulations formed after prolonged overexpression of human mutant tau. Thus, we speculate that this tau pff-injection model may represent a more disease-relevant approach for studying tau aggregation than the traditional tau $\mathrm{Tg}$ mouse overexpression models.

Luk et al. (2012) recently demonstrated that synthetic $\alpha$-synuclein fibrils alone can initiate transmission of PD-like $\alpha$-synuclein pathologies similar to tau fibrils described here when injected into mutant $\alpha$-synuclein Tg mice (M83), implicating shared pathogenic mechanisms underlying neurodegenerative diseases with proteinaceous intraneuronal inclusions. However, several differences emerge from the findings of the two studies. First, despite identical inoculation procedures, injection of $\alpha$-synuclein pffs into striatum and overlaying cortex of M83 mice led to much more widespread $\alpha$-synuclein pathology throughout the brain, including hypothalamus, brainstem, spinal cord, and white matter tracts, than the spread of tau inclusions in PS19 mice that propagated to more restricted and specific brain regions. Second, $\alpha$-synuclein pff-injected M83 mice rapidly succumbed to neurological conditions and eventually death, whereas tau pff injection did not appear to compromise the lifespan of PS19 animals at the ages examined here. This difference is probably attributable to the development of profuse $\alpha$-synuclein inclusions in brainstem and spinal cord in M83 mice but the lack of tau pathology in these areas in PS19 mice. These two closely related studies therefore point to intriguing differences in the spreading pattern of pathological tau and $\alpha$-synuclein. We postulate that the more aggressive progression of $\alpha$-synuclein pathology could be partly attributable to higher propensity of $\alpha$-synuclein to aggregate than tau. In vitro studies have shown that recombinant $\alpha$-synuclein can readily fibrillize on its own, but the fibrillization of recombinant tau usually requires anionic cofactors (Goedert et al., 1996; Chirita et al., 2003; Necula et al., 2003). It is thus conceivable that a very small amount of misfolded seeds transmitted to downstream neurons may be sufficient to template soluble $\alpha$-synuclein aggregation, but this does not occur as readily with tau. Furthermore, pathological $\alpha$-synuclein could spread more readily than pathological tau because of the high concentration of $\alpha$-synuclein at presynaptic terminals, potentially critical sites for interneuronal transmission.

The evidence presented here, that synthetic tau pffs are wholly sufficient to induce and propagate AD-like tau pathology from an inoculation site to widespread interconnected brain regions via neural networks, and in recent studies, that synthetic $\alpha$-synuclein fibrils as well as $\mathrm{A} \beta$ fibrils also can initiate transmission of pathol- ogies (Luk et al., 2012; Stöhr et al., 2012), support the transmissibility of fibrillar species of misfolded proteins. Together, transmission of disease proteins may represent a shared pathogenic mechanism of disease progression underlying multiple neurodegenerative disorders characterized by accumulations of proteinaceous inclusions as initially reported for kuru and other prion diseases.

\section{References}

Aston-Jones G, Cohen JD (2005) An integrative theory of locus coeruleusnorepinephrine function: adaptive gain and optimal performance. Annu Rev Neurosci 28:403-450. CrossRef Medline

Aston-Jones G, Ennis M, Pieribone VA, Nickell WT, Shipley MT (1986) The brain nucleus locus coeruleus: restricted afferent control of a broad efferent network. Science 234:734-737. CrossRef Medline

Ballatore C, Lee VM-Y, Trojanowski JQ (2007) Tau-mediated neurodegeneration in Alzheimer's disease and related disorders. Nat Rev Neurosci 8:663-672. CrossRef Medline

Berridge CW, Waterhouse BD (2003) The locus coeruleus-noradrenergic system: modulation of behavioral state and state-dependent cognitive processes. Brain Res Brain Res Rev 42:33-84. CrossRef Medline

Braak H, Braak E (1991) Neuropathological stageing of Alzheimer-related changes. Acta Neuropathol 82:239-259. CrossRef Medline

Braak H, Del Tredici K (2011a) The pathological process underlying Alzheimer's disease in individuals under thirty. Acta Neuropathol 121:171-181. Medline

Braak H, Del Tredici K (2011b) Alzheimer's pathogenesis: is there neuronto-neuron propagation? Acta Neuropathol 121:589-595. CrossRef Medline

Carroll JC, Iba M, Bangasser DA, Valentino RJ, James MJ, Brunden KR, Lee VM-Y, Trojanowski JQ (2011) Chronic stress exacerbates tau pathology, neurodegeneration, and cognitive performance through a corticotropin-releasing factor receptor-dependent mechanism in a transgenic mouse model of tauopathy. J Neurosci 31:14436-14449. CrossRef Medline

Chan-Palay V (1991) Alterations in the locus coeruleus in dementias of Alzheimer's and Parkinson's disease. Prog Brain Res 88:625-630. CrossRef Medline

Chirita CN, Necula M, Kuret J (2003) Anionic micelles and vesicles induce tau fibrillization in vitro. J Biol Chem 278:25644-25650. CrossRef Medline

Clavaguera F, Bolmont T, Crowther RA, Abramowski D, Frank S, Probst A, Fraser G, Stalder AK, Beibel M, Staufenbiel M, Jucker M, Goedert M, Tolnay M (2009) Transmission and spreading of tauopathy in transgenic mouse brain. Nat Cell Biol 11:909-913. CrossRef Medline

Cohen TJ, Guo JL, Hurtado DE, Kwong LK, Mills IP, Trojanowski JQ, Lee VM-Y (2011) The acetylation of tau inhibits its function and promotes pathological tau aggregation. Nat Commun 2:252. CrossRef Medline

de Calignon A, Polydoro M, Suárez-Calvet M, William C, Adamowicz DH, Kopeikina KJ, Pitstick R, Sahara N, Ashe KH, Carlson GA, Spires-Jones TL, Hyman BT (2012) Propagation of tau pathology in a model of early Alzheimer's disease. Neuron 73:685-697. CrossRef Medline

Drechsel DN, Hyman AA, Cobb MH, Kirschner MW (1992) Modulation of the dynamic instability of tubulin assembly by the microtubule-associated protein tau. Mol Biol Cell 3:1141-1154. Medline

Eisele YS, Obermüller U, Heilbronner G, Baumann F, Kaeser SA, Wolburg H, Walker LC, Staufenbiel M, Heikenwalder M, Jucker M (2010) Peripherally applied Abeta-containing inoculates induce cerebral betaamyloidosis. Science 330:980-982. CrossRef Medline

Foote SL, Bloom FE, Aston-Jones G (1983) Nucleus locus ceruleus: new evidence of anatomical and physiological specificity. Physiol Rev 63:844 914. Medline

Frost B, Jacks RL, Diamond MI (2009) Propagation of tau misfolding from the outside to the inside of a cell. J Biol Chem 284:12845-12852. CrossRef Medline

German DC, Manaye KF, White CL 3rd, Woodward DJ, McIntire DD, Smith WK, Kalaria RN, Mann DM (1992) Disease-specific patterns of locus coeruleus cell loss. Ann Neurol 32:667-676. CrossRef Medline

Goedert M, Jakes R, Spillantini MG, Hasegawa M, Smith MJ, Crowther RA (1996) Assembly of microtubule-associated protein tau into Alzheimerlike filaments induced by sulphated glycosaminoglycans. Nature 383: 550-553. CrossRef Medline 
Gómez-Isla T, Hollister R, West H, Mui S, Growdon JH, Petersen RC, Parisi JE, Hyman BT (1997) Neuronal loss correlates with but exceeds neurofibrillary tangles in Alzheimer's disease. Ann Neurol 41:17-24. CrossRef Medline

Gong CX, Liu F, Grundke-Iqbal I, Iqbal K (2005) Post-translational modifications of tau protein in Alzheimer's disease. J Neural Transm 112:813838. CrossRef Medline

Guo JL, Lee VM-Y (2011) Seeding of normal Tau by pathological Tau conformers drives pathogenesis of Alzheimer-like tangles. J Biol Chem 286: 15317-15331. CrossRef Medline

Hurtado DE, Molina-Porcel L, Iba M, Aboagye AK, Paul SM, Trojanowski JQ, Lee VM-Y (2010) A $\{$ beta\} accelerates the spatiotemporal progression of tau pathology and augments tau amyloidosis in an Alzheimer mouse model. Am J Pathol 177:1977-1988. CrossRef Medline

Hutton M, Lendon CL, Rizzu P, Baker M, Froelich S, Houlden H, PickeringBrown S, Chakraverty S, Isaacs A, Grover A, Hackett J, Adamson J, Lincoln S, Dickson D, Davies P, Petersen RC, Stevens M, de Graaff E, Wauters E, van Baren J, et al (1998) Association of missense and 5'-splice-site mutations in tau with the inherited dementia FTDP-17. Nature 393:702705. CrossRef Medline

Irwin DJ, Cohen TJ, Grossman M, Arnold SE, Xie SX, Lee VM-Y, Trojanowski JQ (2012) Acetylated tau, a novel pathological signature in Alzheimer's disease and other tauopathies. Brain 135:807-818. CrossRef Medline

Kfoury N, Holmes BB, Jiang H, Holtzman DM, Diamond MI (2012) Transcellular propagation of Tau aggregation by fibrillar species. J Biol Chem 287:19440-19451. CrossRef Medline

Lasagna-Reeves CA, Castillo-Carranza DL, Sengupta U, Guerrero-Munoz MJ, Kiritoshi T, Neugebauer V, Jackson GR, Kayed R (2012) Alzheimer brain-derived tau oligomers propagate pathology from endogenous tau. Sci Rep 2:700. CrossRef Medline

Lee VM-Y, Balin BJ, Otvos L Jr, Trojanowski JQ (1991) A68: a major subunit of paired helical filaments and derivatized forms of normal Tau. Science 251:675-678. CrossRef Medline

Lee VM-Y, Goedert M, Trojanowski JQ (2001) Neurodegenerative tauopathies. Annu Rev Neurosci 24:1121-1159. CrossRef Medline

Lee VM-Y, Brunden KR, Hutton M, Trojanowski JQ (2011) Developing therapeutic approaches to tau, selected kinases, and related neuronal protein targets. Cold Spring Harb Perspect Med 1:a006437. CrossRef Medline

Liu L, Drouet V, Wu JW, Witter MP, Small SA, Clelland C, Duff K (2012) Trans-synaptic spread of tau pathology in vivo. PLoS One 7:e31302. CrossRef Medline

Luk KC, Kehm VM, Zhang B, O’Brien P, Trojanowski JQ, Lee VM-Y (2012) Intracerebral inoculation of pathological alpha-synuclein initiates a rapidly progressive neurodegenerative alpha-synucleinopathy in mice. J Exp Med 209:975-986. CrossRef Medline
Marien MR, Colpaert FC, Rosenquist AC (2004) Noradrenergic mechanisms in neurodegenerative diseases: a theory. Brain Res Brain Res Rev 45:38-78. CrossRef Medline

Martin L, Latypova X, Terro F (2011) Post-translational modifications of tau protein: implications for Alzheimer's disease. Neurochem Int 58:458 471. CrossRef Medline

Meyer-Luehmann M, Coomaraswamy J, Bolmont T, Kaeser S, Schaefer C, Kilger E, Neuenschwander A, Abramowski D, Frey P, Jaton AL, Vigouret JM, Paganetti P, Walsh DM, Mathews PM, Ghiso J, Staufenbiel M, Walker LC, Jucker M (2006) Exogenous induction of cerebral betaamyloidogenesis is governed by agent and host. Science 313:1781-1784. CrossRef Medline

Min SW, Cho SH, Zhou Y, Schroeder S, Haroutunian V, Seeley WW, Huang EJ, Shen Y, Masliah E, Mukherjee C, Meyers D, Cole PA, Ott M, Gan L (2010) Acetylation of tau inhibits its degradation and contributes to tauopathy. Neuron 67:953-966. CrossRef Medline

Necula M, Chirita CN, Kuret J (2003) Rapid anionic micelle-mediated alpha-synuclein fibrillization in vitro. J Biol Chem 278:46674-46680. CrossRef Medline

Paxinos G, Franklin KJ (2003) The mouse brain in stereotaxic coordinates, Ed 2. Waltham, MA: Academic.

Spillantini MG, Murrell JR, Goedert M, Farlow MR, Klug A, Ghetti B (1998) Mutation in the tau gene in familial multiple system tauopathy with presenile dementia. Proc Natl Acad Sci U S A 95:7737-7741. CrossRef Medline

Stöhr J, Watts JC, Mensinger ZL, Oehler A, Grillo SK, DeArmond SJ, Prusiner SB, Giles K (2012) Purified and synthetic Alzheimer's amyloid beta (Abeta) prions. Proc Natl Acad Sci U S A 109:11025-11030. CrossRef Medline

Terry RD, Peck A, DeTeresa R, Schechter R, Horoupian DS (1981) Some morphometric aspects of the brain in senile dementia of the Alzheimer type. Ann Neurol 10:184-192. CrossRef Medline

Witman GB, Cleveland DW, Weingarten MD, Kirschner MW (1976) Tubulin requires tau for growth onto microtubule initiating sites. Proc Natl Acad Sci U S A 73:4070-4074. CrossRef Medline

Yoshiyama Y, Higuchi M, Zhang B, Huang SM, Iwata N, Saido TC, Maeda J, Suhara T, Trojanowski JQ, Lee VM-Y (2007) Synapse loss and microglial activation precede tangles in a P301S tauopathy mouse model. Neuron 53:337-351. CrossRef Medline

Zhang B, Carroll J, Trojanowski JQ, Yao Y, Iba M, Potuzak JS, Hogan AM, Xie SX, Ballatore C, Smith AB 3rd, Lee VM-Y, Brunden KR (2012) The microtubule-stabilizing agent, epothilone $\mathrm{d}$, reduces axonal dysfunction, neurotoxicity, cognitive deficits, and Alzheimer-like pathology in an interventional study with aged tau transgenic mice. J Neurosci 32:36013611. CrossRef Medline 